\title{
INVOLUTIONS AND THE FRICKE SPACES OF SURFACES WITH BOUNDARY
}

\author{
Hong Chan Kim
}

\begin{abstract}
The purpose of this paper is to find expressions of the Fricke spaces of some basic surfaces which are a three-holed sphere $\Sigma(0,3)$, a one-holed torus $\Sigma(1,1)$, and a four-holed sphere $\Sigma(0,4)$. For this goal, we define the involutions corresponding to oriented axes of loxodromic elements and an inner product $\langle$,$\rangle which gives the information about$ locations of axes of loxodromic elements. The signs of traces of holonomy elements, which are calculated by lifting a representation from $\mathbf{P S L}(2, \mathbb{C})$ to $\mathbf{S L}(2, \mathbb{C})$, play a very important role in determining the discreteness of holonomy groups.
\end{abstract}

\section{Introduction}

The study of $\mathbf{P S L}(2, \mathbb{C})$-character variety of surfaces is quite active in various areas of topology and geometry such as Kleinian groups, the topological quantum field theory, complex and real projective structures, and the Fricke spaces. In particular, the algebraic properties of matrices in $\mathbf{P S L}(2, \mathbb{C})$ give a close relationship between algebra and geometry. Roughly speaking, the $\operatorname{PSL}(2, \mathbb{C})$-character variety of a smooth surface $M$ is the space of representations of $\pi_{1}(M)$ into $\operatorname{PSL}(2, \mathbb{C})$ up to conjugation.

A hyperbolic structure on a smooth surface $M$ is a representation of $M$ as a quotient $\Omega / \Gamma$ of a convex domain $\Omega \subset \mathbb{H}^{2}$ by a discrete group $\Gamma \subset \mathbf{P S L}(2, \mathbb{R})$ acting properly and freely. Let $M=\Sigma(g, n)$ be a compact connected oriented surface with $g$-genus and $n$-boundary components. Suppose $M$ has non-empty boundary. If the Euler characteristic $\chi(M)=2-2 g-n$ of $M$ is negative, then $M$ admits a hyperbolic structure with geodesic boundary. The deformation space of hyperbolic structures on $M$ is called the Fricke space of $M$. The Fricke space is often identified with the Teichüller space because the uniformization theorem identifies the hyperbolic structures with the conformal structures on

Received September 3, 2013; Revised September 16, 2013.

2010 Mathematics Subject Classification. 57M05, 22E40, $20 \mathrm{H} 10$.

Key words and phrases. Fricke space, involution, discrete holonomy group.

The author gratefully acknowledges the support from a Korea University Grant. 
$M$. Since a hyperbolic structure on $M$ produces a discrete faithful homomorphism

$$
h: \pi_{1}(M) \rightarrow \operatorname{PSL}(2, \mathbb{R}) \subset \mathbf{P S L}(2, \mathbb{C})
$$

up to conjugation, the Fricke space $\mathcal{F}(M)$ can be considered as a subspace of the PSL $(2, \mathbb{C})$-character variety of $M$.

The purpose of this paper is to find expressions of the Fricke spaces of some basic surfaces which are a three-holed sphere $\Sigma(0,3)$, a one-holed torus $\Sigma(1,1)$, and a four-holed sphere $\Sigma(0,4)$. To do these, we define the involutions corresponding to the oriented axes of loxodromic elements of $\mathbf{S L}(2, \mathbb{C})$ and denote the collection of such involutions by $\mathcal{I} n v$. And we also define an inner product $\langle$,$\rangle on \mathcal{I} n v$ which gives the information about locations of axes of loxodromic and hyperbolic elements. During these processes, we shall find matrix representations of discrete holonomy groups of surfaces.

In Section 2, we recall some preliminary definitions about the character varieties and the Fricke spaces of surfaces. In Section 3, we define the sign of non-zero complex numbers, and the involution corresponding to the oriented axis of a loxodromic element. From these we present relations between axes of loxodromic elements. In Sections 4, we calculate the expressions of the Fricke spaces of $\Sigma(0,3), \Sigma(1,1)$, and $\Sigma(0,4)$ by the values of coordinate characters which are trace functions on $\mathbf{S L}(2, \mathbb{C})$-character variety.

\section{Preliminaries}

\subsection{Character variety}

Let $M$ be a smooth manifold. We denote the set of all representations of $\pi_{1}(M)$ into $\mathbf{S L}(2, \mathbb{C})$ by $\mathcal{R}_{S L}(M)$. Then $\mathcal{R}_{S L}(M)$ is an affine algebraic set because $\mathbf{S L}(2, \mathbb{C})$ is an affine algebraic group. The group $\mathbf{S L}(2, \mathbb{C})$ acts on $\mathcal{R}_{S L}(M)$ by conjugation. The algebraic quotient

$$
\mathcal{X}_{S L}(M)=\mathcal{R}_{S L}(M) / / \mathbf{S L}(2, \mathbb{C})
$$

is called the $\mathbf{S L}(2, \mathbb{C})$ - character variety of $M$; i.e., the points in $\mathcal{X}_{S L}(M)$ are the equivalent classes of $\mathcal{R}_{S L}(M)$ which are the closures of orbits under $\mathbf{S L}(2, \mathbb{C})$ conjugation. A representation $\rho: \pi_{1}(M) \rightarrow \mathbf{S L}(2, \mathbb{C})$ is called irreducible if $\rho\left(\pi_{1}(M)\right)$ fixes no point of $\mathbb{C P}^{1}$. If we restrict $\mathcal{R}_{S L}(M)$ to the set of irreducible representations $\mathcal{R}_{S L}^{\prime}(M)$, then the algebraic quotient $\mathcal{X}_{S L}^{\prime}(M)$ is the usual quotient by the action of $\mathbf{S L}(2, \mathbb{C})$ and $\mathcal{X}_{S L}^{\prime}(M)$ is a Zariski dense open subset of $\mathcal{X}_{S L}(M)$. Procesi's paper [12] says that the ring of invariants on $\mathcal{R}_{S L}(M)$ is generated by characters

$$
\rho \stackrel{t_{\gamma}}{\longmapsto} \operatorname{tr}(\rho(\gamma))
$$

where $\rho \in \mathcal{R}_{S L}(M)$ and $\gamma \in \pi_{1}(M)$. By results of Magnus [9], the $\mathbf{S L}(2, \mathbb{C})$ character variety is determined by the values of some characters. 
Similarly the PSL $(2, \mathbb{C})$-character variety of $M$ is defined. The set of all representations of $\pi_{1}(M)$ into $\mathbf{P S L}(2, \mathbb{C})$ is denoted by $\mathcal{R}_{P S L}(M)$. The algebraic quotient $\mathcal{X}_{P S L}(M)$ is called the PSL $(2, \mathbb{C})$-character variety of $M$. A representation $\rho: \pi_{1}(M) \rightarrow \mathbf{P S L}(2, \mathbb{C})$ is called irreducible if $\rho\left(\pi_{1}(M)\right)$ fixes no point of $\mathbb{C P}^{1}$.

\subsection{Lifting representations from $\operatorname{PSL}(2, \mathbb{C})$ to $\operatorname{SL}(2, \mathbb{C})$}

Since the trace is only defined on $\mathbf{S L}(2, \mathbb{C})$, we need the conditions which ensure that a representation into $\mathbf{P S L}(2, \mathbb{C})$ lifts to $\mathbf{S L}(2, \mathbb{C})$. In general, the canonical map $\mathcal{R}_{S L}(M) \rightarrow \mathcal{R}_{P S L}(M)$ is not surjective since there may exist a $\mathbf{P S L}(2, \mathbb{C})$-representation which does not lift to a $\mathbf{S L}(2, \mathbb{C})$-representation.

A PSL $(2, \mathbb{C})$-representation lifts to a $\mathbf{S L}(2, \mathbb{C})$-representation if and only if the second Stiefel-Whitney class $w_{2}(\rho) \in H^{2}(M ; \mathbb{Z} / 2 \mathbb{Z})$ vanishes (Culler [2]). Therefore if $M$ is a surface with boundary or equivalently $\pi_{1}(M)$ is a free group, then every PSL $(2, \mathbb{C})$-representation can be lifted to $\mathbf{S L}(2, \mathbb{C})$-representation since lifting each generator suffices to define a lifted representation. For a closed surface $M$ of genus $g>1$, Goldman [4] showed that $\mathcal{R}_{P S L}(M)$ has exactly two components, one is the set of liftable representations and the other is the set of non-liftable representations.

If a $\mathbf{P S L}(2, \mathbb{C})$-representation lifts, then any other lift is obtained by the action of $H^{1}(M ; \mathbb{Z} / 2 \mathbb{Z})$, which is isomorphic to $\operatorname{Hom}\left(\pi_{1}(M), \mathbb{Z} / 2 \mathbb{Z}\right)$. Thus we regard an element of $H^{1}(M ; \mathbb{Z} / 2 \mathbb{Z})$ as a function $\epsilon: \pi_{1}(M) \rightarrow\{ \pm 1\}$ such that $\epsilon$ acts on $\rho$ by $(\epsilon \cdot \rho)(\gamma)=\epsilon(\gamma) \rho(\gamma)$ for $\gamma \in \pi_{1}(M)$ (Morgan and Shalen [11]).

For example, suppose $M$ is a three-holed sphere (or a pair of pants) $\Sigma(0,3)$. Then $H^{1}(M ; \mathbb{Z} / 2 \mathbb{Z}) \cong \operatorname{Hom}\left(\pi_{1}(M), \mathbb{Z} / 2 \mathbb{Z}\right)$ is isomorphic to $\mathbb{Z} / 2 \mathbb{Z} \oplus \mathbb{Z} / 2 \mathbb{Z}$. Thus if $\left\{A_{1}, A_{2}, A_{3}\right\}$ with $A_{3} A_{2} A_{1}=I$ is a lifted $\mathbf{S L}(2, \mathbb{C})$-representation of $\Sigma(0,3)$, then

$$
\left\{-A_{1},-A_{2}, A_{3}\right\},\left\{A_{1},-A_{2},-A_{3}\right\} \text {, and }\left\{-A_{1}, A_{2},-A_{3}\right\}
$$

are other liftable $\mathbf{S L}(2, \mathbb{C})$-representations. Consider another example $M=$ $\Sigma(1,1)$ a one-holed torus. Then $H^{1}(M ; \mathbb{Z} / 2 \mathbb{Z})$ is isomorphic to $\mathbb{Z} / 2 \mathbb{Z} \oplus \mathbb{Z} / 2 \mathbb{Z}$. Thus if $\{A, B, C\}$ with $C B^{-1} A^{-1} B A=I$ is a lifted $\mathbf{S L}(2, \mathbb{C})$-representation of $\Sigma(1,1)$, then

$$
\{-A, B, C\},\{A,-B, C\} \text {, and }\{-A,-B, C\}
$$

are other liftable $\mathbf{S L}(2, \mathbb{C})$-representations.

\subsection{Fricke spaces}

Our main object is the deformation space of hyperbolic structures on a compact oriented surface $M=\Sigma(g, n)$ with geodesic boundary. Such deformation space is called the Fricke space by Bers-Gardiner [1].

For a given hyperbolic structure on $M$, the action of $\pi_{1}(M)$ on the universal covering space $\tilde{M}$ produces a homomorphism $h: \pi_{1}(M) \rightarrow \mathbf{P S L}(2, \mathbb{R})$ called 
the holonomy homomorphism and it is well-defined up to conjugation. The the holonomy homomorphism induces the holonomy map

$$
\text { hol : } \mathcal{F}(M) \longrightarrow \mathcal{X}_{P S L(2, \mathbb{R})}^{\prime}(M) \subset \mathcal{X}_{P S L}^{\prime}(M)
$$

which is an embedding onto a connected open subset of the irreducible realcharacter variety of dimension $6 g-6+3 n$ (Goldman [3]). Therefore the Fricke space $\mathcal{F}(M)$ is diffeomorphic to $\mathbb{R}^{6 g-6+3 n}$.

\section{Matrices and involutions}

\subsection{Matrices with the fixed points $x$ and $y$}

Recall that $\mathbf{S L}(2, \mathbb{C})$ acts on the projective space $\mathbb{C P}^{1}$. Let $A$ be an element of $\mathbf{S L}(2, \mathbb{C})$ with the eigenvalues $\lambda$ and $\lambda^{-1}$. We denote by $x$ the fixed point of $A$ corresponding to the eigenvalue $\lambda$; i.e., $x \in \mathbb{C P}^{1}$ is the projective class of the eigenvectors of $A$ corresponding to $\lambda$. Another fixed point of $A$ corresponding to $\lambda^{-1}$ is denoted by $y$. If $|\lambda|>1$, then $x$ is the attracting fixed point and $y$ is the repelling fixed point of $A$. Suppose $A$ has two distinct fixed points. Then the matrix $A$ is uniquely determined by $\lambda, x$ and $y$ as follows. Suppose $x$ and $y$ are not infinite. The relations $A\left(\begin{array}{c}x \\ 1\end{array}\right)=\left(\begin{array}{c}\lambda x \\ \lambda\end{array}\right)$ and $A\left(\begin{array}{c}y \\ 1\end{array}\right)=\left(\begin{array}{c}\lambda^{-1} y \\ \lambda^{-1}\end{array}\right)$ induce $A\left(\begin{array}{cc}x & y \\ 1 & 1\end{array}\right)=\left(\begin{array}{cc}\lambda x & \lambda^{-1} y \\ \lambda & \lambda^{-1}\end{array}\right)$. Therefore the matrix $A=A_{(\lambda, x, y)}$ is

$$
A_{(\lambda, x, y)}=\frac{1}{x-y}\left(\begin{array}{cc}
\lambda x-\lambda^{-1} y & -\left(\lambda-\lambda^{-1}\right) x y \\
\lambda-\lambda^{-1} & \lambda^{-1} x-\lambda y
\end{array}\right) .
$$

We can also calculate

(4)

$$
A_{(\lambda, \infty, y)}=\left(\begin{array}{cc}
\lambda & -\left(\lambda-\lambda^{-1}\right) y \\
0 & \lambda^{-1}
\end{array}\right) \text { and } A_{(\lambda, x, \infty)}=\left(\begin{array}{cc}
\lambda^{-1} & \left(\lambda-\lambda^{-1}\right) x \\
0 & \lambda
\end{array}\right) .
$$

And we can easily show that $A_{\left(\lambda^{-1}, y, x\right)}=A_{(\lambda, x, y)}$ and $A_{(\lambda, x, y)}^{-1}=A_{(\lambda, y, x)}$.

\subsection{Involutions}

We are interested in the projective involutions and involutions of $\mathbb{C P}^{1}$. We define a projective involution of $\mathbb{C P}^{1}$ is a projective transformation in $\operatorname{PSL}(2, \mathbb{C})$ of order two. An involution of $\mathbb{C P}^{1}$ is a transformation $\xi$ in $\mathbf{S L}(2, \mathbb{C})$ such that $\xi^{2}$ is a scalar matrix but $\xi$ is not.

Proposition 3.1. Let $\xi \in \mathbf{S L}(2, \mathbb{C})$. Then the followings are equivalent:

(1) $\xi$ is an involution.

(2) $\xi^{2}=-I$.

(3) $\operatorname{tr}(\xi)=0$.

(4) $\xi$ is conjugate to $\left(\begin{array}{cc}i & 0 \\ 0 & -i\end{array}\right)$.

Proof. (1) $\Rightarrow(2)$ Suppose $\xi^{2}=k I$. Since $1^{2}=\operatorname{det}(\xi)^{2}=\operatorname{det}\left(\xi^{2}\right)=k^{2}$, we have $\xi^{2}= \pm I$. If $\xi^{2}=I$, then $\operatorname{det}(\xi)=1$ implies $\xi= \pm I$, a contradiction. (2) $\Rightarrow(3)$ From $\xi^{2}-\operatorname{tr}(\xi) \xi+I=0$, we get $\operatorname{tr}(\xi)=0$. (3) $\Rightarrow$ (4) Since $\operatorname{det}(\xi)=1, \xi$ has 
reciprocal eigenvalues $\lambda$ and $\lambda^{-1}$. The condition $\operatorname{tr}(A)=0$ induces eigenvalues are $i$ and $-i$. (4) $\Rightarrow$ (1) Obvious.

We denote the collection of involutions in $\mathbf{S L}(2, \mathbb{C})$ by

$$
\mathcal{I} n v:=\mathbf{S L}(2, \mathbb{C}) \cap \mathfrak{s l}(2, \mathbb{C})=\{\xi \in \mathbf{S L}(2, \mathbb{C}) \mid \operatorname{tr}(\xi)=0\}
$$

where $\mathfrak{s l}(2, \mathbb{C})$ is the set of traceless $2 \times 2$ matrices. The quotient

$$
\mathbb{P}(\mathcal{I} n v):=\operatorname{I} n v /( \pm I) \subset \operatorname{PSL}(2, \mathbb{C})
$$

consists of all projective involutions of $\mathbb{C P}^{1}$.

Proposition 3.2. Suppose $x$ and $y$ are two distinct points in $\mathbb{C P}^{1}$. Then the projective involution in $\mathbf{P S L}(2, \mathbb{C})$ fixing $x$ and $y$ is

$$
\pm \frac{i}{x-y}\left(\begin{array}{cc}
x+y & -2 x y \\
2 & -x-y
\end{array}\right) \stackrel{\text { let }}{=} \mathbb{P}\left(\xi_{(x, y)}\right) \text {. }
$$

If $x=\infty($ or $y=\infty)$, then the corresponding projective involution is

(6) $\pm i\left(\begin{array}{cc}1 & -2 y \\ 0 & -1\end{array}\right) \stackrel{\text { let }}{=} \mathbb{P}\left(\xi_{(\infty, y)}\right) \quad\left(\right.$ or $\left.\pm i\left(\begin{array}{cc}-1 & 2 x \\ 0 & 1\end{array}\right) \stackrel{\text { let }}{=} \mathbb{P}\left(\xi_{(x, \infty)}\right)\right)$.

Proof. Eigenvalues of each involution are $i$ and $-i$. Therefore we get $\mathbb{P}\left(\xi_{(x, y)}\right)$, $\mathbb{P}\left(\xi_{(\infty, y)}\right)$ and $\mathbb{P}\left(\xi_{(x, \infty)}\right)$ by plugging in $\lambda= \pm i$ for the matrices (3) and (4).

Since $\mathbb{P}\left(\xi_{(x, y)}\right)=\mathbb{P}\left(\xi_{(y, x)}\right)$, it is natural $\mathbb{P}(\mathcal{I} n v)$ identifies with the collection of unordered pairs of distinct points in $\mathbb{C P}^{1}$. We will interpret $\mathcal{I} n v$ as the collection of ordered pairs of distinct points in $\mathbb{C P}^{1}$.

\subsection{3-dimensional hyperbolic geometry}

We use the upper half space model $\mathbb{H}^{3}$ as follows. The algebra of Hamiltonian quaternion is the $\mathbb{R}$-algebra generated by $1, i, j, k$ subject to the relations $i^{2}=$ $j^{2}=k^{2}=-1, i j=-j i, j k=-k j$ and $k i=-i k$. The upper half space model $\mathbb{H}^{3}$ is defined by

$$
\mathbb{H}^{3}:=\{z+u j \mid z \in \mathbb{C}, u \in \mathbb{R}, u>0\} .
$$

The Lie group $\mathbf{S L}(2, \mathbb{C})$ acts on $\mathbb{H}^{3}$ by

$$
\left(\begin{array}{ll}
a & b \\
c & d
\end{array}\right) \cdot(z+u j)=(a(z+u j)+b)(c(z+u j)+d)^{-1} .
$$

The elements of $\mathbf{S L}(2, \mathbb{C})$ are classified into three different types (Ratcliffe [13]). We classify non-central elements (i.e., $A \neq \pm I$ ) by their eigenvalues and traces. Suppose $\lambda$ is an eigenvalue of $A$ such that $|\lambda| \geq 1$;

- $A$ is elliptic $\Leftrightarrow A$ fixes a point in $\mathbb{H}^{3} \Leftrightarrow|\lambda|=1$ and $\lambda \neq \pm 1 \Leftrightarrow$ $\operatorname{tr}(A) \in(-2,2)$.

- $A$ is parabolic $\Leftrightarrow A$ fixes no point in $\mathbb{H}^{3}$ and fixes a unique point in $\mathbb{C P}^{1}$ $\Leftrightarrow \lambda= \pm 1 \Leftrightarrow \operatorname{tr}(A)= \pm 2$. 
- $A$ is loxodromic $\Leftrightarrow A$ fixes no point in $\mathbb{H}^{3}$ and fixes two points in $\mathbb{C P}^{1}$ $\Leftrightarrow|\lambda|>1 \Leftrightarrow \operatorname{tr}(A) \in \mathbb{C} \backslash[-2,2]$.

Let $A$ be an element of $\mathbf{S L}(2, \mathbb{C})$ such that $A \neq \pm I$. Then the following conditions are equivalent:

- $A$ has two distinct eigenvalues.

- $\operatorname{tr}(A) \neq \pm 2$.

- $A$ has two distinct fixed points in $\mathbb{C P}^{1}$.

- $A$ leaves invariant a unique geodesic $\ell_{A}$ in $\mathbb{H}^{3}$, each of whose endpoints is fixed.

The corresponding transformation of $\mathbb{H}^{3}$ is either elliptic or loxodromic. The unique invariant geodesic of $A$ is called the axis of $A$. If $A$ is elliptic, then the set of fixed points of $A$ is exactly the axis $\ell_{A}$ of $A$.

Suppose $A \in \mathbf{S L}(2, \mathbb{C})$ has two distinct fixed points in $\mathbb{C P}^{1}$. We are going to find the projective involution $\mathbb{P}\left(\xi_{A}\right) \in \mathbf{P S L}(2, \mathbb{C})$ such that $A$ and $\mathbb{P}\left(\xi_{A}\right)$ have the same fixed points. Let $A^{\prime}=2 A-\operatorname{tr}(A) I$. Then $\operatorname{tr}\left(A^{\prime}\right)=0$ and $A^{\prime} A=A A^{\prime}$; i.e. $A^{\prime}$ is a traceless matrix which commutes with $A$. Since $A$ has two distinct fixed points, $\operatorname{tr}(A) \neq \pm 2$. Thus $\operatorname{det}\left(A^{\prime}\right)=4-\operatorname{tr}(A)^{2} \neq 0$. For a non-parabolic element $A=\left(\begin{array}{ll}a & b \\ c & d\end{array}\right) \in \mathbf{S L}(2, \mathbb{C})$, we define

$$
\mathbb{P}\left(\xi_{A}\right):= \pm \frac{i(2 A-\operatorname{tr}(A) I)}{\sqrt{\operatorname{tr}(A)^{2}-4}}= \pm \frac{i}{\sqrt{(a+d)^{2}-4}}\left(\begin{array}{cc}
a-d & 2 b \\
2 c & d-a
\end{array}\right) .
$$

If $A$ is an involution in $\mathbf{S L}(2, \mathbb{C})$, then we obtain $\mathbb{P}\left(\xi_{A}\right)= \pm A$. If $A$ is not an involution, then $\mathbb{P}\left(\xi_{A}\right) A=A \mathbb{P}\left(\xi_{A}\right)$ induces the fixed points of $\mathbb{P}\left(\xi_{A}\right)$ and $A$ are the same. Thus $\mathbb{P}\left(\xi_{A}\right)$ is the projective involution in $\operatorname{PSL}(2, \mathbb{C})$.

\subsection{Matrix representations for oriented geodesics in $\mathbb{H}^{3}$}

Geodesics in $\mathbb{H}^{3}$ correspond to unordered pairs of distinct points in $\mathbb{C P}^{1}$ via their endpoints. Thus a geodesic $\ell \subset \mathbb{H}^{3}$ corresponds uniquely to the projective involution $\mathbb{P}\left(\xi_{\ell}\right) \in \mathbf{P S L}(2, \mathbb{C})$ such that the fixed points in $\mathbb{C P}^{1}$ are the endpoints of $\ell$. Oriented geodesics correspond to ordered pairs of distinct points in $\mathbb{C P}^{1}$. We will represent oriented geodesics in $\mathbb{H}^{3}$ by involutions in $\mathbf{S L}(2, \mathbb{C})$ as follows. Suppose $\ell$ is an oriented geodesic in $\mathbb{H}^{3}$. Let $x$ be the attracting endpoint and $y$ the repelling endpoint of $\ell$. By (5) and (6), we can find involutions $\xi_{\ell}$ and $-\xi_{\ell} \in \mathbf{S L}(2, \mathbb{C})$ which fix $x$ and $y$. Since eigenvalues of each involution are distinct, $\xi_{\ell}$ has 1-dimensional eigenspaces corresponding to $i$ and $-i$. These eigenspaces determine the fixed points in $\mathbb{C P}^{1}$. Exchanging $\xi_{\ell}$ with $-\xi_{\ell}$ interchanges the $\pm i$-eigenspaces. In this way, we identify the collection of oriented geodesics in $\mathbb{H}^{3}$ with the space of involutions $\mathcal{I} n v \subset \mathbf{S L}(2, \mathbb{C})$.

Definition. The $(-i)$-eigenspace of an involution in $\mathcal{I} n v$ corresponds to the repelling endpoint of an oriented geodesic in $\mathbb{H}^{3}$. Another $i$-eigenspace corresponds to the attracting endpoint of an oriented geodesic. 
For example, the involution $\left(\begin{array}{cc}i & 0 \\ 0 & -i\end{array}\right)$ represents the oriented geodesic from 0 to $\infty$, since $\left(\begin{array}{l}0 \\ 1\end{array}\right)$ and $\left(\begin{array}{l}1 \\ 0\end{array}\right)$ are the eigenvectors of $\left(\begin{array}{cc}i & 0 \\ 0 & -i\end{array}\right)$ corresponding to the eigenvalues $-i$ and $i$ respectively. Similarly $\left(\begin{array}{cc}-i & 0 \\ 0 & i\end{array}\right)$ represents the oriented geodesic from $\infty$ to 0 .

Suppose $A$ is a loxodromic element of $\mathbf{S L}(2, \mathbb{C})$. Then the axis of $A$ has the orientation from the repelling fixed point to the attracting fixed point. To determine the involution $\xi_{A} \in \mathcal{I} n v$ corresponding to a loxodromic element $A \in \mathbf{S L}(2, \mathbb{C})$, we need to define the sign of $z \in \mathbb{C}$. We choose the negative real axis for a branch cut of $\arg z$; i.e. $\arg z \in(-\pi, \pi]$ for a nonzero $z$ in $\mathbb{C}$. Then we can define the root of $z$ as a single valued function by

$$
\sqrt{z}:=\sqrt{r} e^{i \theta / 2}
$$

for $z=r e^{i \theta}$ with $r>0$ and $\theta \in(-\pi, \pi]$.

Definition. For a nonzero complex number $z$, the sign of $z$ is defined by $\operatorname{sgn}(z)=1$ if $\arg (z) \in\left(-\frac{\pi}{2}, \frac{\pi}{2}\right]$ and $\operatorname{sgn}(z)=-1$ otherwise.

For any nonzero real number $x \in \mathbb{R}, \operatorname{sgn}(x)=1$ if $x>0$ and $\operatorname{sgn}(x)=-1$ if $x<0$. And we have $\operatorname{sgn}(x) x=|x|=\sqrt{x^{2}}$. By the definition of $\operatorname{sign}, \operatorname{sgn}(i)=1$ and $\operatorname{sgn}(-i)=-1$. Thus $\sqrt{i^{2}}=\sqrt{-1}=i=\operatorname{sgn}(i) i$ and $\sqrt{(-i)^{2}}=\sqrt{-1}=i=$ $-(-i)=\operatorname{sgn}(-i)(-i)$. Generally we have the following Proposition 3.3.

Proposition 3.3. $\sqrt{z^{2}}=\operatorname{sgn}(z) z$ for any nonzero $z$ in $\mathbb{C}$.

Proof. If $\arg (z)=\theta \in\left(-\frac{\pi}{2}, \frac{\pi}{2}\right]$, then $\arg \left(z^{2}\right)=2 \theta \in(-\pi, \pi]$. Thus $\sqrt{z^{2}}=$ $\sqrt{r^{2}} e^{i(2 \theta / 2)}=r e^{i \theta}=z=\operatorname{sgn}(z) z$. If $\arg (z)=\theta \in\left(\frac{\pi}{2}, \pi\right]$, then $\arg \left(z^{2}\right)=$ $2 \theta-2 \pi \in(-\pi, 0]$. Thus $\sqrt{z^{2}}=\sqrt{r^{2}} e^{i(2 \theta-2 \pi) / 2}=r e^{i \theta}(-1)=-z=\operatorname{sgn}(z) z$. Similarly we can show $\sqrt{z^{2}}=-z=\operatorname{sgn}(z) z$ for $\arg (z)=\theta \in\left(-\pi,-\frac{\pi}{2}\right]$.

Now we can determine the involution $\xi_{A} \in \mathcal{I} n v$ corresponding to a loxodromic element $A \in \mathbf{S L}(2, \mathbb{C})$.

Theorem 3.4. Suppose $A \in \mathbf{S L}(2, \mathbb{C})$ is a loxodromic element with the oriented axis $\ell_{A}$. Then the involution $\xi_{A} \in \mathcal{I} n v$ corresponding to $A$ (or the axis $\ell_{A}$ ) is

$$
\xi_{A}=\frac{\varepsilon i(2 A-\operatorname{tr}(A) I)}{\sqrt{\operatorname{tr}(A)^{2}-4}}=\frac{\varepsilon i}{\sqrt{(a+d)^{2}-4}}\left(\begin{array}{cc}
a-d & 2 b \\
2 c & d-a
\end{array}\right)
$$

where $\varepsilon=\operatorname{sgn}(\operatorname{tr}(A))$.

Proof. Suppose $\lambda$ is the eigenvalue of a loxodromic element $A$ such that $|\lambda|>1$. Let $x$ and $y$ be the attracting and repelling fixed points of $A$ respectively. If $x$ and $y$ are finite, then the loxodromic transformation $A$ is expressed by the matrix $A_{(\lambda, x, y)}$ in (3). Thus $\xi_{A}$ is

$$
\xi_{A}=\frac{\varepsilon i}{\sqrt{\left(\lambda+\lambda^{-1}\right)^{2}-4}} \frac{\left(\lambda-\lambda^{-1}\right)}{(x-y)}\left(\begin{array}{cc}
x+y & -2 x y \\
2 & -x-y
\end{array}\right)
$$




$$
\begin{aligned}
& =\frac{\varepsilon i\left(\lambda-\lambda^{-1}\right)}{\sqrt{\left(\lambda-\lambda^{-1}\right)^{2}}} \frac{1}{(x-y)}\left(\begin{array}{cc}
x+y & -2 x y \\
2 & -x-y
\end{array}\right) \\
& =\frac{\varepsilon i}{\operatorname{sgn}\left(\lambda-\lambda^{-1}\right)} \frac{1}{(x-y)}\left(\begin{array}{cc}
x+y & -2 x y \\
2 & -x-y
\end{array}\right) .
\end{aligned}
$$

Since

$$
\xi_{A}\left(\begin{array}{c}
x \\
1
\end{array}\right)=\frac{\varepsilon i}{\operatorname{sgn}\left(\lambda-\lambda^{-1}\right)}\left(\begin{array}{c}
x \\
1
\end{array}\right) \text { and } \xi_{A}\left(\begin{array}{l}
y \\
1
\end{array}\right)=\frac{\varepsilon(-i)}{\operatorname{sgn}\left(\lambda-\lambda^{-1}\right)}\left(\begin{array}{l}
y \\
1
\end{array}\right)
$$

$\xi_{A}$ is the involution corresponding to the oriented geodesic $\ell_{A}$ if and only if $x$ and $y$ are the eigenspaces corresponding to the eigenvalues $i$ and $-i$ respectively if and only if $\varepsilon=\operatorname{sgn}\left(\lambda-\lambda^{-1}\right)$. Let $\lambda=r e^{i \theta}$, then

$$
\lambda+\lambda^{-1}=\left(r+r^{-1}\right) \cos \theta+i\left(r-r^{-1}\right) \sin \theta
$$

and

$$
\lambda-\lambda^{-1}=\left(r-r^{-1}\right) \cos \theta+i\left(r+r^{-1}\right) \sin \theta .
$$

Since $r=|\lambda|>1$, two nonzero complex numbers $\lambda-\lambda^{-1}$ and $\operatorname{tr}(A)=\lambda+\lambda^{-1}$ are contained in the same quadrant. Therefore $\operatorname{sgn}\left(\lambda-\lambda^{-1}\right)=\operatorname{sgn}(\operatorname{tr}(A))$. We can prove similarly for the cases $x=\infty$ or $y=\infty$. It completes the proof.

Corollary 3.5. Let $\xi_{A}$ be the involution in $\mathcal{I}_{n} v$ corresponding to a loxodromic element $A \in \mathbf{S L}(2, \mathbb{C})$. Then $\xi_{-A}=\xi_{A}$ and $\xi_{A^{-1}}=-\xi_{A}$.

Proof. We denote $\varepsilon_{A}=\operatorname{sgn}(\operatorname{tr}(A))$. Then we have $\varepsilon_{-A}=-\varepsilon_{A}$. Thus

$$
\xi_{-A}=\frac{(-\varepsilon) i(2(-A)-\operatorname{tr}(-A) I)}{\sqrt{\operatorname{tr}(-A)^{2}-4}}=\frac{(-1)^{2} \varepsilon i(2 A-\operatorname{tr}(A) I)}{\sqrt{\operatorname{tr}(A)^{2}-4}}=\xi_{A} .
$$

From $A^{2}-\operatorname{tr}(A) A+I=0$, we induce $2 A-2 \operatorname{tr}(A) I+2 A^{-1}=0$. Thus

$$
\xi_{A^{-1}}=\frac{\varepsilon i\left(2 A^{-1}-\operatorname{tr}\left(A^{-1}\right) I\right)}{\sqrt{\operatorname{tr}\left(A^{-1}\right)^{2}-4}}=\frac{\varepsilon i(-2 A+\operatorname{tr}(A) I)}{\sqrt{\operatorname{tr}(A)^{2}-4}}=-\xi_{A}
$$

since $2 A^{-1}-\operatorname{tr}\left(A^{-1}\right) I=2 A^{-1}-\operatorname{tr}(A) I=\operatorname{tr}(A) I-2 A$.

Remark 3.6. If we define the involution $\xi_{A}$ without the $\operatorname{sign}$ of $\operatorname{tr}(A)$ (i.e., $\left.\xi_{A}=\frac{i(2 A-\operatorname{tr}(A) I)}{\sqrt{\operatorname{tr}(A)^{2}-4}}\right)$, then $\xi_{-A}$ becomes $-\xi_{A}$. Thus $\varepsilon=\operatorname{sgn}(\operatorname{tr}(A))$ is essential to define $\xi_{A}$ since $A$ and $-A$ have exactly the same oriented axes. The second relation represents the orientation $\ell_{A^{-1}}$ is opposite to that of $\ell_{A}$.

For example, let $A=\left(\begin{array}{cc}\mu & 0 \\ 0 & \mu^{-1}\end{array}\right)$ be a loxodromic element. Then the involution corresponding to $A$ is

$$
\xi_{A}=\frac{\operatorname{sgn}\left(\mu+\mu^{-1}\right)}{\operatorname{sgn}\left(\mu-\mu^{-1}\right)}\left(\begin{array}{cc}
i & 0 \\
0 & -i
\end{array}\right) .
$$

Thus $\xi_{A}=\left(\begin{array}{cc}i & 0 \\ 0 & -i\end{array}\right)$ if $|\mu|>1$ and $\xi_{A}=\left(\begin{array}{cc}-i & 0 \\ 0 & i\end{array}\right)$ if $0<|\mu|<1$. 


\subsection{2-dimensional hyperbolic geometry}

We define the hyperbolic plane $\mathbb{H}^{2}$ by

$$
\mathbb{H}^{2}:=\left\{z+u j \in \mathbb{H}^{3} \mid z \in \mathbb{R}\right\} .
$$

Then a transformation $A \in \mathbf{S L}(2, \mathbb{C})$ preserves $\mathbb{H}^{2}$ if and only if $A \in \mathbf{S L}(2, \mathbb{R})$ or $A \in \mathbf{S L}(2, i \mathbb{R})$; i.e., $A$ is a real matrix of determinant 1 or $A$ is a purelyimaginary matrix of determinant 1 . The first case is an orientation-preserving transformation of $\mathbb{H}^{2}$ and the second case is orientation-reversing.

Suppose $\ell$ is an oriented geodesic in $\mathbb{H}^{2}$ with the attracting endpoint $x$ and the repelling endpoint $y$. Then the corresponding involutions is

$$
\xi_{(x, y)}=\frac{i}{x-y}\left(\begin{array}{cc}
x+y & -2 x y \\
2 & -x-y
\end{array}\right)
$$

by Proposition 3.2 and Theorem 3.4. Since $x, y \in \mathbb{R P}^{1}, \xi_{(x, y)}$ is a purelyimaginary matrix. Therefore we can identify the space of oriented geodesics in $\mathbb{H}^{2}$ with the collection of involutions in $\mathbf{S L}(2, i \mathbb{R})$, that is

$$
\{\xi \in \mathbf{S L}(2, i \mathbb{R}) \mid \operatorname{tr}(\xi)=0\} \subset \mathcal{I} n v .
$$

\subsection{Inner product of $\mathfrak{s l}(2, \mathbb{R})$}

We think of $\mathfrak{s l}(2, \mathbb{R})$ as a 3 -dimensional space with the signature $(2,1)$ as follows. Let $\mathbb{R}^{2,1}$ be the 3 -dimensional space with the $(2,1)$-signature inner product such that

$$
\langle v, w\rangle=v_{1} w_{1}+v_{2} w_{2}-v_{3} w_{3} .
$$

The function $\Phi: \mathbb{R}^{2,1} \rightarrow \mathfrak{s l}(2, \mathbb{R})$ defined by

$$
\Phi(v)=\Phi\left(v_{1}, v_{2}, v_{3}\right)=\left(\begin{array}{cc}
v_{1} & v_{2}-v_{3} \\
v_{2}+v_{3} & -v_{1}
\end{array}\right)
$$

produces an equivariant $(2,1)$-signature inner product of $\mathfrak{s l}(2, \mathbb{R})$ such that

$$
\langle\xi, \eta\rangle:=\left\langle\Phi^{-1}(\xi), \Phi^{-1}(\eta)\right\rangle
$$

for $\xi, \eta \in \mathfrak{s l}(2, \mathbb{R})$.

Proposition 3.7. Let $\xi, \eta \in \mathfrak{s l}(2, \mathbb{R})$. Then

$$
\langle\xi, \eta\rangle=\frac{1}{2} \operatorname{tr}(\xi \eta) .
$$

is a $(2,1)$-signature inner product of $\mathfrak{s l}(2, \mathbb{R})$.

Proof. Since the inverse $\Phi^{-1}: \mathfrak{s l}(2, \mathbb{R}) \rightarrow \mathbb{R}^{2,1}$ is

$$
\Phi^{-1}\left(\begin{array}{cc}
a & b \\
c & -a
\end{array}\right)=\left(a, \frac{1}{2}(c+b), \frac{1}{2}(c-b)\right),
$$

we can show $\left\langle\Phi^{-1}(\xi), \Phi^{-1}(\eta)\right\rangle=\frac{1}{2} \operatorname{tr}(\xi \eta)$ through some computations. 


\subsection{Locations of axes}

A loxodromic element $A$ in $\mathbf{S L}(2, \mathbb{R})$ is called hyperbolic. Thus a hyperbolic element $A$ is an orientation-preserving transformation of $\mathbb{H}^{2}$ such that $A$ has exactly two fixed points on $\partial \mathbb{H}^{2}=\mathbb{R P}^{1}$. The axis of a hyperbolic element $A$ is an oriented geodesic in $\mathbb{H}^{2}$. Thus there exists the corresponding involution $\xi_{A} \in \mathbf{S L}(2, i \mathbb{R})$ by Theorem 3.4. Since $\operatorname{tr}(A)>2$ or $\operatorname{tr}(A)<-2$ for a hyperbolic element $A$, the sign of $\operatorname{tr}(A)$ is 1 if $\operatorname{tr}(A)$ is positive and -1 otherwise.

We say two distinct geodesics in $\mathbb{H}^{2}$ are crossing if they intersect in $\mathbb{H}^{2}$, asymptotic if they do not intersect in $\mathbb{H}^{2}$ but one of their endpoints are the same, and separated if the closures of geodesics do not intersect in $\mathbb{H}^{2} \cup \partial \mathbb{H}^{2}$. Given an oriented geodesic $\ell$ in $\mathbb{H}^{2}$, we define a well-determined open halfplane in $\mathbb{H}^{2}$, which is bounded by $\ell$. The choice of half-plane $\mathcal{H}_{\ell} \subset \mathbb{H}^{2} \backslash \ell$ is the righthand-side half-plane when we walk along the oriented geodesic $\ell$.

Definition. Suppose two oriented geodesics $\ell_{1}$ and $\ell_{2}$ in $\mathbb{H}^{2}$ are asymptotic or separated. We say $\ell_{1}$ and $\ell_{2}$ are with the same direction if the half-planes $\mathcal{H}_{\ell_{1}} \cap \mathcal{H}_{\ell_{2}}=\emptyset$ or $\mathcal{H}_{\ell_{1}} \cup \mathcal{H}_{\ell_{2}}=\mathbb{H}^{2}$. $\ell_{1}$ and $\ell_{2}$ are with the opposite direction if $\mathcal{H}_{\ell_{1}} \subset \mathcal{H}_{\ell_{2}}$ or $\mathcal{H}_{\ell_{2}} \subset \mathcal{H}_{\ell_{1}}$.

We extend the inner product of $\mathfrak{s l}(2, \mathbb{R})$ to the space of involutions $\mathcal{I} n v$ which is $\mathfrak{s l}(2, \mathbb{C}) \cap \mathbf{S L}(2, \mathbb{C})$. For involutions $\xi, \eta \in \mathcal{I} n v$, we define

$$
\langle\xi, \eta\rangle:=\frac{1}{2} \operatorname{tr}(\xi \eta)
$$

Then the value $\langle\xi, \eta\rangle$ represents the locations of oriented geodesics in $\mathbb{H}^{3}$ corresponding to $\xi$ and $\eta$.

Theorem 3.8. Suppose $A_{1}, A_{2} \in \mathbf{S L}(2, \mathbb{R})$ are hyperbolic elements with distinct oriented axes $\ell_{1}, \ell_{2}$ in $\mathbb{H}^{2}$ respectively. Let $\xi_{1}, \xi_{2}$ be corresponding involutions in $\mathbf{S L}(2, i \mathbb{R})$ to the axes $\ell_{1}, \ell_{2}$ respectively. Then the following conditions are equivalent:

(1) $\left\langle\xi_{1}, \xi_{2}\right\rangle>1 \Longleftrightarrow \ell_{1}, \ell_{2}$ are separated with the same direction.

(2) $\left\langle\xi_{1}, \xi_{2}\right\rangle<-1 \Longleftrightarrow \ell_{1}, \ell_{2}$ are separated with the opposite direction.

(3) $\left\langle\xi_{1}, \xi_{2}\right\rangle=1 \Longleftrightarrow \ell_{1}, \ell_{2}$ are asymptotic with the same direction.

(4) $\left\langle\xi_{1}, \xi_{2}\right\rangle=-1 \Longleftrightarrow \ell_{1}, \ell_{2}$ are asymptotic with the opposite direction.

(5) $-1<\left\langle\xi_{1}, \xi_{2}\right\rangle<1 \Longleftrightarrow \ell_{1}, \ell_{2}$ are crossing.

(6) $\left\langle\xi_{1}, \xi_{2}\right\rangle=0 \Longleftrightarrow \ell_{1}, \ell_{2}$ are orthogonal.

Proof. Let $B=P A P^{-1}$. Then $\xi_{B}=P \xi_{A} P^{-1}$. Thus the value $\langle\xi, \eta\rangle$ is a conjugacy invariant. Without loss of generality, we may assume $A_{1}$ is a diagonal matrix $\left(\begin{array}{cc}\lambda & 0 \\ 0 & \lambda^{-1}\end{array}\right)$ with $\lambda^{2}>1$. Suppose $x$ and $y$ are the attracting and repelling fixed points of $A_{2}$, respectively. Then we have

$$
\xi_{1}=\left(\begin{array}{cc}
i & 0 \\
0 & -i
\end{array}\right) \text { and } \xi_{2}=\frac{i}{x-y}\left(\begin{array}{cc}
x+y & -2 x y \\
2 & -x-y
\end{array}\right)
$$


from (10). Thus

$$
\left\langle\xi_{1}, \xi_{2}\right\rangle=\frac{1}{2} \operatorname{tr}\left(\xi_{1} \xi_{2}\right)=\frac{y+x}{y-x} .
$$

(1) Recall that $\ell_{1}$ is the oriented geodesic from 0 to $\infty$. Then oriented geodesics $\ell_{1}, \ell_{2}$ are separated with the same direction $\Leftrightarrow y<x<0$ or $0<x<y \Leftrightarrow$ $x(y-x)>0 \Leftrightarrow \frac{y+x}{y-x}>1$. (2) We can similarly show that $\ell_{1}, \ell_{2}$ are separated with the opposite direction $\Leftrightarrow x<y<0$ or $0<y<x \Leftrightarrow y(y-x)<0 \Leftrightarrow$ $\frac{y+x}{y-x}<-1$. (3) $\ell_{1}, \ell_{2}$ are asymptotic with the same direction $\Leftrightarrow x=0$ or $y=\infty$ $\Leftrightarrow\left\langle\xi_{1}, \xi_{2}\right\rangle=1$. (4) $\ell_{1}, \ell_{2}$ are asymptotic with the opposite direction $\Leftrightarrow y=0$ or $x=\infty \Leftrightarrow\left\langle\xi_{1}, \xi_{2}\right\rangle=-1$. (5) $\ell_{1}, \ell_{2}$ are crossing if and only if $x<0<y$ or $y<0<x \Leftrightarrow x y<0 \Leftrightarrow(y+x)^{2}<(y-x)^{2} \Leftrightarrow\left(\frac{y+x}{y-x}\right)^{2}<1$. (6) $\ell_{1}, \ell_{2}$ are orthogonal $\Leftrightarrow x=-y \Leftrightarrow \frac{y+x}{y-x}=0$.

For loxodromic elements in $\mathbf{S L}(2, \mathbb{C})$, we have the following similar results.

Corollary 3.9. Suppose $A_{1}, A_{2} \in \mathbf{S L}(2, \mathbb{C})$ are loxodromic elements with distinct oriented axes $\ell_{1}, \ell_{2}$ in $\mathbb{H}^{3}$ respectively. Let $\xi_{1}, \xi_{2}$ be corresponding involutions in $\mathcal{I} n v$ to the axes $\ell_{1}, \ell_{2}$ respectively. Then the following conditions are equivalent:

(1) $\left\langle\xi_{1}, \xi_{2}\right\rangle \in(-1,1) \Longleftrightarrow \ell_{1}, \ell_{2}$ are crossing.

(2) $\left\langle\xi_{1}, \xi_{2}\right\rangle=0 \Longleftrightarrow \ell_{1}, \ell_{2}$ are orthogonal.

(3) $\left\langle\xi_{1}, \xi_{2}\right\rangle=1 \Longleftrightarrow \ell_{1}, \ell_{2}$ are asymptotic with the same direction.

(4) $\left\langle\xi_{1}, \xi_{2}\right\rangle=-1 \Longleftrightarrow \ell_{1}, \ell_{2}$ are asymptotic with the opposite direction.

(5) $\left\langle\xi_{1}, \xi_{2}\right\rangle \in \mathbb{C} \backslash[-1,1] \Longleftrightarrow \ell_{1}, \ell_{2}$ are separated.

Proof. Use the same assumption in the proof of Theorem 3.8. Without loss of generality, we may assume the axis $\ell_{1}$ is the oriented geodesic from 0 to $\infty$. Note that if $x$ and $y$ are the fixed points of $A_{2}$, then $P(x)$ and $P(y)$ are fixed points of $P A_{2} P^{-1}$. For the cases $(1) \sim(4)$, there exists a rotation $P \in \mathbf{S L}(2, \mathbb{C})$ around the axis $\ell_{1}$ such that the fixes points of $P A_{2} P^{-1}$ lie in $\partial \mathbb{H}^{2}$. Thus the axis of $P A_{2} P^{-1}$ is contained in $\mathbb{H}^{2}$. Since the value $\left\langle\xi_{1}, \xi_{2}\right\rangle$ is a conjugacy invariant, we have the same results in Theorem 3.8 for cases $(1) \sim(4)$. The case (5) is obvious by contraposition.

The value $\left\langle\xi_{1}, \xi_{2}\right\rangle$ can be calculated from the traces and signs of matrices.

Proposition 3.10. Suppose $A_{1}, A_{2} \in \mathbf{S L}(2, \mathbb{C})$ are loxodromic elements. Let $\xi_{j}$ be the involution in $\mathcal{I} n v$ corresponding to $A_{j}$. Then

$$
\left\langle\xi_{1}, \xi_{2}\right\rangle=\frac{\varepsilon_{1} \varepsilon_{2}\left(t_{1} t_{2}-2 t_{12}\right)}{\sqrt{t_{1}^{2}-4} \sqrt{t_{2}^{2}-4}}
$$

where $\varepsilon_{j}=\operatorname{sgn}\left(\operatorname{tr}\left(A_{j}\right)\right), t_{j}=\operatorname{tr}\left(A_{j}\right)$ and $t_{12}=\operatorname{tr}\left(A_{1} A_{2}\right)$. 
Proof. From Theorem 3.4, we know

$$
\xi_{j}=\frac{\varepsilon_{j} i}{\sqrt{\operatorname{tr}\left(A_{j}\right)^{2}-4}}\left(2 A_{j}-\operatorname{tr}\left(A_{j}\right) I\right)=\frac{\varepsilon_{j} i}{\sqrt{t_{j}^{2}-4}}\left(2 A_{j}-t_{j} I\right) .
$$

Thus

$$
\begin{aligned}
& \left\langle\xi_{1}, \xi_{2}\right\rangle \\
= & \frac{1}{2} \operatorname{tr}\left(\xi_{1} \xi_{2}\right) \\
= & \frac{\varepsilon_{1} \varepsilon_{2} i^{2}}{2 \sqrt{t_{1}^{2}-4} \sqrt{t_{2}^{2}-4}} \operatorname{tr}\left(4 A_{1} A_{2}-2 t_{2} A_{1}-2 t_{1} A_{2}+t_{1} t_{2} I\right) \\
= & \frac{-\varepsilon_{1} \varepsilon_{2}}{2 \sqrt{t_{1}^{2}-4} \sqrt{t_{2}^{2}-4}}\left\{4 \operatorname{tr}\left(A_{1} A_{2}\right)-2 t_{2} \operatorname{tr}\left(A_{1}\right)-2 t_{1} \operatorname{tr}\left(A_{2}\right)+t_{1} t_{2} \operatorname{tr}(I)\right\} \\
= & \frac{-\varepsilon_{1} \varepsilon_{2}}{2 \sqrt{t_{1}^{2}-4} \sqrt{t_{2}^{2}-4}}\left\{4 t_{12}-2 t_{2} t_{1}-2 t_{1} t_{2}+2 t_{1} t_{2}\right\}=\frac{\varepsilon_{1} \varepsilon_{2}\left(t_{1} t_{2}-2 t_{12}\right)}{\sqrt{t_{1}^{2}-4} \sqrt{t_{2}^{2}-4}} .
\end{aligned}
$$

In the following Corollary 3.11, we know that the relations between axes $\ell_{1}$ and $\ell_{2}$ are completely determined by signs and traces of corresponding matrices. In this case, the sign of the function

$$
f_{12}:=t_{1}^{2}+t_{2}^{2}+t_{12}^{2}-t_{1} t_{2} t_{12}-4
$$

is important.

Corollary 3.11. Let $A_{1}, A_{2} \in \mathbf{S L}(2, \mathbb{R})$ be hyperbolic elements and $\xi_{j}$ the involution corresponding to $A_{j}$. Then the following conditions are equivalent:

(1) $\left\langle\xi_{1}, \xi_{2}\right\rangle>1 \Longleftrightarrow \varepsilon_{1} \varepsilon_{2}\left(t_{1} t_{2}-2 t_{12}\right)>0$ and $f_{12}>0$.

(2) $\left\langle\xi_{1}, \xi_{2}\right\rangle=1 \Longleftrightarrow \varepsilon_{1} \varepsilon_{2}\left(t_{1} t_{2}-2 t_{12}\right)>0$ and $f_{12}=0$.

(3) $-1<\left\langle\xi_{1}, \xi_{2}\right\rangle<1 \Longleftrightarrow f_{12}<0$.

(4) $\left\langle\xi_{1}, \xi_{2}\right\rangle=-1 \Longleftrightarrow \varepsilon_{1} \varepsilon_{2}\left(t_{1} t_{2}-2 t_{12}\right)<0$ and $f_{12}=0$.

(5) $\left\langle\xi_{1}, \xi_{2}\right\rangle<-1 \Longleftrightarrow \varepsilon_{1} \varepsilon_{2}\left(t_{1} t_{2}-2 t_{12}\right)<0$ and $f_{12}>0$,

where $f_{12}=t_{1}^{2}+t_{2}^{2}+t_{12}^{2}-t_{1} t_{2} t_{12}-4$.

Proof. (1) From Proposition 3.10, $\left\langle\xi_{1}, \xi_{2}\right\rangle>1$ is equivalent to

$$
\begin{aligned}
& \varepsilon_{1} \varepsilon_{2}\left(t_{1} t_{2}-2 t_{12}\right)>\sqrt{t_{1}^{2}-4} \sqrt{t_{2}^{2}-4} \\
\Leftrightarrow \quad & \varepsilon_{1} \varepsilon_{2}\left(t_{1} t_{2}-2 t_{12}\right)>0 \text { and }\left(t_{1} t_{2}-2 t_{12}\right)^{2}>\left(t_{1}^{2}-4\right)\left(t_{2}^{2}-4\right) \\
\Leftrightarrow \quad & \varepsilon_{1} \varepsilon_{2}\left(t_{1} t_{2}-2 t_{12}\right)>0 \text { and } t_{1}^{2}+t_{2}^{2}+t_{12}^{2}-t_{1} t_{2} t_{12}-4=f_{12}>0 .
\end{aligned}
$$

Similarly we can calculate others.

From results of Corollary 3.11 and Theorem 3.8, we obtain the following relations;

$$
\begin{aligned}
& f_{12}>0 \quad \Longleftrightarrow \quad \ell_{1} \text { and } \ell_{2} \text { are separated } \\
& f_{12}=0 \Longleftrightarrow \ell_{1} \text { and } \ell_{2} \text { are asymptotic }
\end{aligned}
$$




$$
f_{12}<0 \Longleftrightarrow \ell_{1} \text { and } \ell_{2} \text { are crossing. }
$$

\section{Fricke spaces of surfaces}

Recall $M=\Sigma(g, n)$ is a compact connected oriented surface with $g$-genus and $n$-boundary components. The Fricke space $\mathcal{F}(M)$ of $M$ is the isotopy classes of hyperbolic structures on $M$ with geodesic boundary.

Let $X$ be a smooth manifold and $G$ an algebraic Lie group. For a general $(G, X)$-structure on $M$, the holonomy homomorphism $h: \pi_{1}(M) \rightarrow G$ is locally injective. It may be not injective (Sullivan and Thurston [14], Weil [15]). For the real projective structure, the holonomy homomorphism $h: \pi_{1}(M) \rightarrow$ $\operatorname{PGL}(n, \mathbb{R})$ is generally not injective. But if the real projective structure is convex, then $h$ is injective (Goldman [5]). Since the convex real projective structures are an extension of the hyperbolic structures (Kim [7]), the holonomy homomorphism $h$ is injective for the hyperbolic structures. Thus the holonomy homomorphism $h: \pi_{1}(M) \rightarrow \mathbf{P S L}(2, \mathbb{R})$ is an isomorphism onto its image $\Gamma$ called the holonomy group. Hence we identify the fundamental group $\pi_{1}(M)$ with the holonomy group $\Gamma$.

Giving a hyperbolic structure on $M$ is equivalent to finding a discrete embedding $h: \pi \rightarrow \mathbf{P S L}(2, \mathbb{R})$ up to conjugation since a faithful holonomy homomorphism induces a developing map into $\mathbb{H}^{2}$ (Matsuzaki and Taniguchi [10], Goldman [6]).

In this section, we will find the Fricke spaces of a three-holed sphere $\Sigma(0,3)$, a one-holed torus $\Sigma(1,1)$, and a four-holed sphere $\Sigma(0,4)$. And we will present an expression of generators of the holonomy group of each surfaces in terms of $\mathbf{S L}(2, \mathbb{R})$ instead of $\mathbf{P S L}(2, \mathbb{R})$. Since we only deal with surfaces with boundary, the lift of a representation from $\operatorname{PSL}(2, \mathbb{R})$ to $\mathbf{S L}(2, \mathbb{R})$ is always possible.

\subsection{Three-holed sphere $\Sigma(0,3)$}

Suppose $M=\Sigma(0,3)$ is a three-holed sphere with boundary components $A_{1}, A_{2}, A_{3}$ subject to the relation

$$
A_{3} A_{2} A_{1}=I .
$$

Suppose $M$ is equipped with a hyperbolic structure. Then every nontrivial element of holonomy group is hyperbolic due to Kuiper [8]. Thus $A_{1}, A_{2}, A_{3} \in$ $\mathbf{S L}(2, \mathbb{R})$ with $\operatorname{tr}\left(A_{j}\right)^{2}>4$ for each $j$.

Theorem 4.1. Suppose $A_{1}, A_{2}, A_{3} \in \mathbf{S L}(2, \mathbb{R})$ are hyperbolic elements such that $A_{3} A_{2} A_{1}=I$. Let $\xi_{j}$ be the involution in $\mathcal{I} n v$ corresponding to $A_{j}$ and $\varepsilon_{j}=\operatorname{sgn}\left(\operatorname{tr}\left(A_{j}\right)\right)$. Then $\left\langle\xi_{1}, \xi_{2}\right\rangle>1,\left\langle\xi_{2}, \xi_{3}\right\rangle>1,\left\langle\xi_{3}, \xi_{1}\right\rangle>1$ if and only if $\varepsilon_{1} \varepsilon_{2} \varepsilon_{3}=-1$.

Proof. Since $A_{3} A_{2} A_{1}=I$, we have

$$
t_{12}=\operatorname{tr}\left(A_{1} A_{2}\right)=\operatorname{tr}\left(A_{2} A_{1}\right)=\operatorname{tr}\left(A_{3}^{-1}\right)=\operatorname{tr}\left(A_{3}\right)=t_{3} .
$$


By Corollary 3.11-(1), $\left\langle\xi_{1}, \xi_{2}\right\rangle>1$ is equivalent to

$$
\varepsilon_{1} \varepsilon_{2}\left(t_{1} t_{2}-2 t_{3}\right)>0 \text { and } t_{1}^{2}+t_{2}^{2}+t_{3}^{2}-t_{1} t_{2} t_{3}-4>0 .
$$

By symmetry, we get $\left\langle\xi_{1}, \xi_{2}\right\rangle>1,\left\langle\xi_{2}, \xi_{3}\right\rangle>1,\left\langle\xi_{3}, \xi_{1}\right\rangle>1$ if and only if

$$
\varepsilon_{i} \varepsilon_{j}\left(t_{i} t_{j}-2 t_{k}\right)>0
$$

for distinct indices $i, j, k$, and

$$
t_{1}^{2}+t_{2}^{2}+t_{3}^{2}-t_{1} t_{2} t_{3}-4>0 .
$$

$(\Leftarrow)$ Suppose $\varepsilon_{1} \varepsilon_{2} \varepsilon_{3}=-1$. Then $t_{j}^{2}=\operatorname{tr}\left(A_{j}\right)^{2}>4$ and $t_{1} t_{2} t_{3}<-8$. Thus $t_{1}^{2}+t_{2}^{2}+t_{3}^{2}-t_{1} t_{2} t_{3}-4>4+4+4+8-4>0$. And $\varepsilon_{i} \varepsilon_{j}\left(t_{i} t_{j}-2 t_{k}\right)=$ $\left(\varepsilon_{i} t_{i}\right)\left(\varepsilon_{j} t_{j}\right)-2\left(\varepsilon_{i} \varepsilon_{j} \varepsilon_{k}\right)\left(\varepsilon_{k} t_{k}\right)=\left|t_{i}\right|\left|t_{j}\right|+2\left|t_{k}\right|>0$. Therefore Equations (16) and (17) hold.

$(\Rightarrow)$ Suppose Equation (16) holds. Then it is equivalent to

$$
\left|t_{i}\right|\left|t_{j}\right|>2\left(\varepsilon_{1} \varepsilon_{2} \varepsilon_{3}\right)\left|t_{k}\right| \text {. }
$$

The value $\varepsilon_{1} \varepsilon_{2} \varepsilon_{3}$ is 1 or -1 . We will show that if $\varepsilon_{1} \varepsilon_{2} \varepsilon_{3}=1$, then Equation (17) is not true. Therefore $\varepsilon_{1} \varepsilon_{2} \varepsilon_{3}$ must be -1 . Without loss of generality, we assume $2<\left|t_{1}\right| \leq\left|t_{2}\right| \leq\left|t_{3}\right|$. If $\varepsilon_{1} \varepsilon_{2} \varepsilon_{3}=1$, then Equation (18) becomes

$$
\left|t_{1}\right|\left|t_{2}\right|>2\left|t_{3}\right| \text {. }
$$

Since we have the relation $\left(\left|t_{1}\right|-\left|t_{2}\right|\right)^{2}<\left(\left|t_{3}\right|-2\right)^{2}$, we obtain

$$
\begin{aligned}
\left(\left|t_{1}\right|-\left|t_{2}\right|\right)^{2}+\left|t_{3}\right|^{2} & <\left(\left|t_{3}\right|-2\right)^{2}+\left|t_{3}\right|^{2}=2\left|t_{3}\right|\left(\left|t_{3}\right|-2\right)+4 \\
& <\left|t_{1}\right|\left|t_{2}\right|\left(\left|t_{3}\right|-2\right)+4=\left|t_{1}\right|\left|t_{2}\right|\left|t_{3}\right|-2\left|t_{1}\right|\left|t_{2}\right|+4 .
\end{aligned}
$$

Thus $t_{1}^{2}+t_{2}^{2}+t_{3}^{2}-t_{1} t_{2} t_{3}-4=t_{1}^{2}+t_{2}^{2}+t_{3}^{2}-\left(\varepsilon_{1} \varepsilon_{2} \varepsilon_{3}\right) t_{1} t_{2} t_{3}-4=\left|t_{1}\right|^{2}+\left|t_{2}\right|^{2}+$ $\left|t_{3}\right|^{2}-\left|t_{1}\right|\left|t_{2}\right|\left|t_{3}\right|-4<0$. This contradicts Equation (17).

From Theorem 3.8, we know $\left\langle\xi_{1}, \xi_{2}\right\rangle>1,\left\langle\xi_{2}, \xi_{3}\right\rangle>1$, and $\left\langle\xi_{3}, \xi_{1}\right\rangle>1$ is equivalent to all axes of $A_{1}, A_{2}, A_{3}$ are mutually separated with the same direction. In this case, we will show that the axes of $A_{1}, A_{2}, A_{3}$ are located as in Figure 1 up to conjugation.

Proposition 4.2. Suppose $A_{1}, A_{2}, A_{3} \in \mathbf{S L}(2, \mathbb{R})$ are hyperbolic elements such that $A_{3} A_{2} A_{1}=I$. Let $\xi_{j}$ be the involution corresponding to $A_{j}$. If $\left.\left\langle\xi_{1}, \xi_{2}\right\rangle\right\rangle$ $1,\left\langle\xi_{2}, \xi_{3}\right\rangle>1$, and $\left\langle\xi_{3}, \xi_{1}\right\rangle>1$, then the axis of $A_{3}$ is located between the repelling fixed point of $A_{1}$ and the attracting fixed point of $A_{2}$.

Proof. Denote by $x_{i}$ the attracting fixed point of $A_{i}$ and $y_{i}$ the repelling fixed point of $A_{i}$. Then the possible locations of axis of $A_{3}$ are between $y_{2}$ and $x_{1}$ or $y_{1}$ and $x_{2}$. Without loss of generality, we assume the axis of $A_{2}$ is the geodesic from 0 to $\infty$ and the axis of $A_{1}$ is located in the positive real part of $\mathbb{H}^{2}$; i.e., we assume $y_{2}=0, x_{2}=\infty$ and $0<x_{1}<y_{1}$. In the first case, we have $0<x_{3}<y_{3}<x_{1}<y_{1}$. Since $A_{2}(z)>z$ for any $z>0$ and $y_{3}<A_{1}\left(y_{3}\right)<x_{1}$, we get $y_{3}=A_{2} A_{1} A_{3}\left(y_{3}\right)=A_{2}\left(A_{1}\left(y_{3}\right)\right)>A_{1}\left(y_{3}\right)>y_{3}$. Contradiction. Therefore the axis of $A_{3}$ must be located between $y_{1}$ and $x_{2}$. 


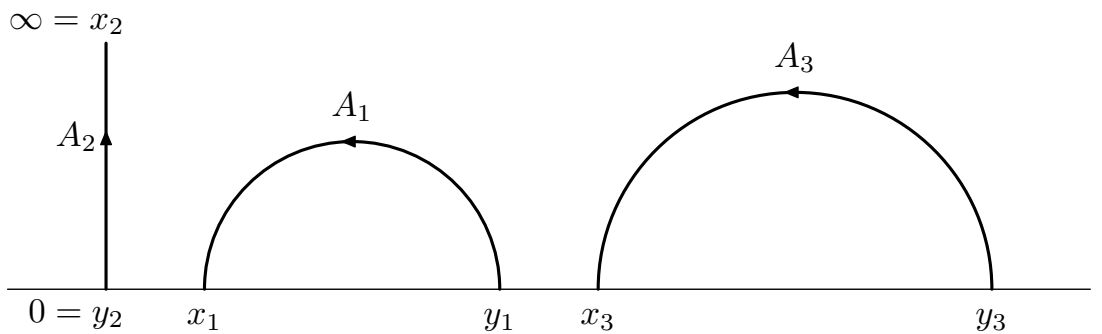

Figure 1. The locations of axes $A_{1}, A_{2}, A_{3}$ with $A_{3} A_{2} A_{1}=I$.

Suppose $A_{1}, A_{2}, A_{3}$ are hyperbolic matrices in $\mathbf{S L}(2, \mathbb{R})$. Then a holonomy group

$$
\Gamma=\left\langle A_{1}, A_{2}, A_{3} \mid A_{3} A_{2} A_{1}=I\right\rangle
$$

of a three-holed sphere $\Sigma(0,3)$ is discrete if and only if the axes of $A_{1}, A_{2}, A_{3}$ are located as in Figure 1 up to conjugation.

The $\mathbf{S L}(2, \mathbb{C})$-character variety $\mathcal{X}_{S L}(M)$ is coordinated by the values of some characters(=trace functions). Since the Fricke space $\mathcal{F}(M)$ is contained in the $\mathbf{P S L}(2, \mathbb{R})$-character variety of $M, \mathcal{F}(M)$ is represented by the values of coordinate traces modulo the action of $H^{1}(M ; \mathbb{Z} / 2 \mathbb{Z})$.

Theorem 4.3. The Fricke space of a three-holed sphere $\Sigma(0,3)$ identifies with the open subset of $\mathbb{R}^{3}$ such that

$$
\left\{\left(t_{1}, t_{2}, t_{3}\right) \in \mathbb{R}^{3} \mid t_{i}<-2\right\}
$$

i.e., $\mathcal{F}(\Sigma(0,3)) \cong(-\infty,-2)^{3}$.

Proof. By the result of Theorem 4.1 and the various of lifts from $\operatorname{PSL}(2, \mathbb{R})$ to $\mathbf{S L}(2, \mathbb{R})$ in (1), the possible traces of $A_{1}, A_{2}, A_{3}$ are

$$
\begin{aligned}
\widetilde{\mathcal{F}}_{0,3} & \stackrel{\text { let }}{=}(2, \infty) \times(2, \infty) \times(-\infty,-2) \bigcup(2, \infty) \times(-\infty,-2) \times(2, \infty) \\
\bigcup & (-\infty,-2) \times(2, \infty) \times(2, \infty) \bigcup(-\infty,-2) \times(-\infty,-2) \times(-\infty,-2) .
\end{aligned}
$$

Thus $\mathcal{F}(\Sigma(0,3))=\widetilde{\mathcal{F}}_{0,3} / H^{1}(M ; \mathbb{Z} / 2 \mathbb{Z}) \cong(-\infty,-2)^{3}$ since points of the Fricke space are considered as representations into $\mathbf{P S L}(2, \mathbb{R})$ up to conjugation.

We give a matrix representation of a discrete holonomy group of a threeholed surface $\Sigma(0,3)$ up to conjugation. By the discreteness, without loss of generality, we assume

$$
A_{1}=\frac{1}{y-x}\left(\begin{array}{cc}
\lambda^{-1} y-\lambda x & \left(\lambda-\lambda^{-1}\right) x y \\
-\left(\lambda-\lambda^{-1}\right) & \lambda y-\lambda^{-1} x
\end{array}\right), \quad A_{2}=\left(\begin{array}{cc}
\mu & 0 \\
0 & \mu^{-1}
\end{array}\right)
$$

with $\lambda<-1, \mu<-1$ and $0<x<y$ (Compare with the matrix in (3)). Then $x$ is the attracting and $y$ is the repelling fixed point of $A_{1}$, respectively since 
$|\lambda|>1$. Let

$$
A_{3}=A_{1}^{-1} A_{2}^{-1}=\frac{1}{y-x}\left(\begin{array}{cc}
\left(\lambda y-\lambda^{-1} x\right) \mu^{-1} & -\left(\lambda-\lambda^{-1}\right) x y \mu \\
\left(\lambda-\lambda^{-1}\right) \mu^{-1} & \left(\lambda^{-1} y-\lambda x\right) \mu
\end{array}\right) .
$$

Then we obtain $A_{3} A_{2} A_{1}=I$ and the trace of $A_{3}$ is

$$
\operatorname{tr}\left(A_{3}\right)=\frac{1}{y-x}\left\{\left(\lambda \mu^{-1}+\lambda^{-1} \mu\right) y-\left(\lambda^{-1} \mu^{-1}+\lambda \mu\right) x\right\} .
$$

We can easily compute

$$
\operatorname{tr}\left(A_{3}\right)=k \Longleftrightarrow \frac{y}{x}=\frac{\lambda \mu+\lambda^{-1} \mu^{-1}-k}{\lambda \mu^{-1}+\lambda^{-1} \mu-k} .
$$

Thus the matrix $A_{3}$ can be all three types; hyperbolic, parabolic and elliptic. For any $k<-2$, if we set

$$
y=\lambda \mu+\lambda^{-1} \mu^{-1}-k \text { and } x=\lambda \mu^{-1}+\lambda^{-1} \mu-k,
$$

then $0<x<y$ and $A_{3}$ becomes a hyperbolic matrix with $\operatorname{tr}\left(A_{3}\right)=k<-2$. Therefore above matrices in (20) and (21) are a representation of a discrete holonomy group of a three-holed surface $\Sigma(0,3)$ because the trace of each matrix is less than -2 . And we also have a condition

$$
\operatorname{tr}\left(A_{3}\right)<-2 \Longleftrightarrow \frac{y}{x}<\left(\frac{\lambda \mu+1}{\lambda+\mu}\right)^{2} .
$$

\subsection{One-holed torus $\Sigma(1,1)$}

Suppose $M=\Sigma(1,1)$ is a one-holed torus with holonomy elements $A, B, C$ subject to the relation

$$
C B^{-1} A^{-1} B A=I \text {. }
$$

The elements $A, B$ correspond to simple closed curves on $\Sigma(1,1)$ which intersect transversely at one point and $C$ corresponds to boundary component.

The following Trace Identity is essential to compute traces of matrices which have some relations. It gives a very useful formula to calculate traces. For example, if matrices $A, B$ and $C$ have the relation $C B^{-1} A^{-1} B A=I$, then we can compute $\operatorname{tr}(C)$ from $\operatorname{tr}(A), \operatorname{tr}(B)$ and $\operatorname{tr}(A B)$.

Proposition 4.4 (Trace Identity). Suppose $A, B \in \mathbf{S L}(2, \mathbb{C})$. Then

$$
\operatorname{tr}(A B)=\operatorname{tr}(A) \operatorname{tr}(B)-\operatorname{tr}\left(A^{-1} B\right) .
$$

Proof. For any $2 \times 2$ matrix $A$, we have the equation

$$
A^{2}-\operatorname{tr}(A) A+\operatorname{det}(A) I=0 .
$$

Let $A, B \in \mathbf{S L}(2, \mathbb{C})$. Right-multiplying Equation $(24)$ by $A^{-1} B$, we obtain $A B-\operatorname{tr}(A) B+A^{-1} B=0$. Therefore $\operatorname{tr}(A B)=\operatorname{tr}(A) \operatorname{tr}(B)-\operatorname{tr}\left(A^{-1} B\right)$.

Remark 4.5. Since $\operatorname{tr}\left(A^{-1} B\right)=\operatorname{tr}\left(B A^{-1}\right)=\operatorname{tr}\left(A B^{-1}\right)$, we also obtain

$$
\operatorname{tr}(A B)=\operatorname{tr}(A) \operatorname{tr}(B)-\operatorname{tr}\left(A B^{-1}\right) .
$$


Proposition 4.6. Suppose $A, B, C \in \mathbf{S L}(2, \mathbb{C})$ with $C B^{-1} A^{-1} B A=I$. Then

$$
\operatorname{tr}(C)=\operatorname{tr}(A)^{2}+\operatorname{tr}(B)^{2}+\operatorname{tr}(A B)^{2}-\operatorname{tr}(A) \operatorname{tr}(B) \operatorname{tr}(A B)-2 .
$$

Proof. Since $C=A^{-1} B^{-1} A B$, we know $\operatorname{tr}(C)=\operatorname{tr}\left(A^{-1} B^{-1} A B\right)$. Thus

$$
\begin{aligned}
\operatorname{tr}(C) & =\operatorname{tr}\left((B A)^{-1} A B\right)=\operatorname{tr}(B A) \operatorname{tr}(A B)-\operatorname{tr}(B A A B) \\
& =\operatorname{tr}(A B)^{2}-\left(\operatorname{tr}(B) \operatorname{tr}\left(A^{2} B\right)-\operatorname{tr}\left(B^{-1} A^{2} B\right)\right) \\
& =\operatorname{tr}(A B)^{2}-\operatorname{tr}(B) \operatorname{tr}\left(A^{2} B\right)+\operatorname{tr}\left(A^{2}\right) \\
& =\operatorname{tr}(A B)^{2}-\operatorname{tr}(B)\left(\operatorname{tr}(A) \operatorname{tr}(A B)-\operatorname{tr}\left(A^{-1} A B\right)\right)+\left(\operatorname{tr}(A)^{2}-\operatorname{tr}(I)\right) \\
& =\operatorname{tr}(A B)^{2}-\operatorname{tr}(B) \operatorname{tr}(A) \operatorname{tr}(A B)+\operatorname{tr}(B)^{2}+\operatorname{tr}(A)^{2}-2 \\
& =\operatorname{tr}(A)^{2}+\operatorname{tr}(B)^{2}+\operatorname{tr}(A B)^{2}-\operatorname{tr}(A) \operatorname{tr}(B) \operatorname{tr}(A B)-2 .
\end{aligned}
$$

by repeatedly applying Trace Identity (23).

For $A, B \in \mathbf{S L}(2, \mathbb{C})$, we define

$$
f_{A B}:=t_{A}^{2}+t_{B}^{2}+t_{A B}^{2}-t_{A} t_{B} t_{A B}-4,
$$

where $t_{A}=\operatorname{tr}(A), t_{B}=\operatorname{tr}(B)$ and $t_{A B}=\operatorname{tr}(A B)$ (Compare with the definition of $f_{12}$ in (14); They are exactly the same). From Proposition 4.6, we induce that the trace of commutator $A B A^{-1} B^{-1}$ is $f_{A B}+2$; i.e.,

$$
\operatorname{tr}\left(A B A^{-1} B^{-1}\right)=f_{A B}+2=t_{A}^{2}+t_{B}^{2}+t_{A B}^{2}-t_{A} t_{B} t_{A B}-2 .
$$

Suppose a one-holed torus $\Sigma(1,1)$ is equipped with a hyperbolic structure; i.e., $A, B, C$ are hyperbolic matrices in $\mathbf{S L}(2, \mathbb{R})$. Then a holonomy group

$$
\Gamma=\left\langle A, B, C \mid C B^{-1} A^{-1} B A=I\right\rangle
$$

of $\Sigma(1,1)$ is discrete if and only if the axes of $A, B, C$ are located as in Figure 2 up to conjugation.

Theorem 4.7. Suppose $A, B, C \in \mathbf{S L}(2, \mathbb{R})$ are hyperbolic elements such that $C B^{-1} A^{-1} B A=I$. Let $\xi_{A}, \xi_{B}, \xi_{C}$ be the involutions corresponding to $A, B, C$ respectively. Let $\varepsilon_{A}=\operatorname{sgn}(\operatorname{tr}(A)), \varepsilon_{B}=\operatorname{sgn}(\operatorname{tr}(B))$ and $\varepsilon_{C}=\operatorname{sgn}(\operatorname{tr}(C))$. Then $\left\langle\xi_{A}, \xi_{B}\right\rangle \in(-1,1),\left\langle\xi_{A}, \xi_{C}\right\rangle>1,\left\langle\xi_{B}, \xi_{C}\right\rangle<-1$ if and only if $\varepsilon_{C}=-1$.

Proof. $(\Leftarrow)$ Suppose $\varepsilon_{C}=-1$. Then $t_{C}=\operatorname{tr}(C)<-2$ since $C$ is hyperbolic. From Equation (25), we know $t_{C}=t_{A}^{2}+t_{B}^{2}+t_{A B}^{2}-t_{A} t_{B} t_{A B}-2$. Thus we have

$$
f_{A B}=t_{A}^{2}+t_{B}^{2}+t_{A B}^{2}-t_{A} t_{B} t_{A B}-4=t_{C}-2<-4<0 .
$$

Therefore $\left\langle\xi_{A}, \xi_{B}\right\rangle \in(-1,1)$ by Corollary 3.11-(3).

Since $A C=B^{-1} A B$, we have $t_{A C}=t_{A}$. Then $\varepsilon_{A} \varepsilon_{C}\left(t_{A} t_{C}-2 t_{A C}\right)=$ $\varepsilon_{A} \varepsilon_{C}\left(t_{A} t_{C}-2 t_{A}\right)=\left|t_{A}\right|\left(\left|t_{C}\right|-2 \varepsilon_{C}\right)=\left|t_{A}\right|\left(\left|t_{C}\right|+2\right)>0$ and

$$
\begin{aligned}
f_{A C} & =t_{A}^{2}+t_{C}^{2}+t_{A C}^{2}-t_{A} t_{C} t_{A C}-4=t_{A}^{2}+t_{C}^{2}+t_{A}^{2}-t_{A} t_{C} t_{A}-4 \\
& =t_{A}^{2}\left(2-t_{C}\right)+\left(t_{C}^{2}-4\right)>0 .
\end{aligned}
$$

Thus $\left\langle\xi_{A}, \xi_{C}\right\rangle>1$ by Corollary 3.11-(1). 
From $C B^{-1}=A^{-1} B^{-1} A$, we obtain $t_{B^{-1} C}=t_{C B^{-1}}=t_{B^{-1}}=t_{B}$. By Trace Identity (23), we have $t_{B C}=t_{B} t_{C}-t_{B{ }^{-1} C}=t_{B} t_{C}-t_{B}$. Then

$$
\begin{aligned}
\varepsilon_{B} \varepsilon_{C}\left(t_{B} t_{C}-2 t_{B C}\right) & =\varepsilon_{B} \varepsilon_{C}\left(t_{B} t_{C}-2 t_{B} t_{C}+2 t_{B}\right) \\
& =\left|t_{B}\right|\left(-\left|t_{C}\right|+2 \varepsilon_{C}\right)=\left|t_{B}\right|\left(-\left|t_{C}\right|-2\right)<0
\end{aligned}
$$

and

$$
\begin{aligned}
f_{B C} & =t_{B}^{2}+t_{C}^{2}+t_{B C}^{2}-t_{B} t_{C} t_{B C}-4 \\
& =t_{B}^{2}+t_{C}^{2}+\left(t_{B} t_{C}-t_{B}\right)^{2}-t_{B} t_{C}\left(t_{B} t_{C}-t_{B}\right)-4 \\
& =t_{B}^{2}\left(2-t_{C}\right)+\left(t_{C}^{2}-4\right)>0 .
\end{aligned}
$$

Thus $\left\langle\xi_{B}, \xi_{C}\right\rangle<-1$ by Corollary 3.11-(5).

$(\Rightarrow)$ Since $\left\langle\xi_{A}, \xi_{B}\right\rangle \in(-1,1)$, we have $f_{A B}=t_{C}-2<0$ by Corollary 3.11-(3). Since $C$ is hyperbolic, we get $t_{C}<-2 \Leftrightarrow \varepsilon_{C}=-1$.

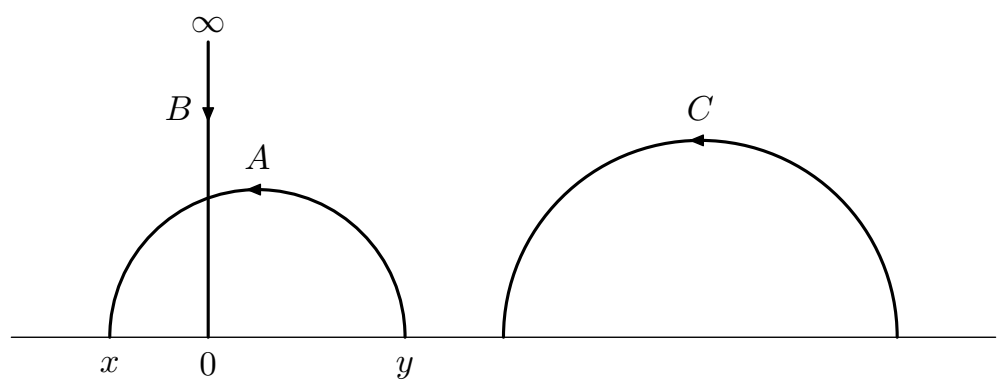

Figure 2. The locations of axes $A, B, C$ with $C B^{-1} A^{-1} B A=I$.

From Theorem 4.7, the only condition for the discreteness of a holonomy group of a one-holed torus is $\operatorname{tr}(C)<-2$. Because $\operatorname{tr}(C)$ is expressed by the values of $\operatorname{tr}(A), \operatorname{tr}(B)$ and $\operatorname{tr}(A B)$, the coordinate traces of the Fricke space of $\Sigma(1,1)$ will be $t_{A}, t_{B}$ and $t_{A B}$.

Proposition 4.8. Suppose $A, B \in \mathbf{S L}(2, \mathbb{R})$ are hyperbolic elements. If the axes of $A$ and $B$ are crossing, then $A B$ is hyperbolic and $\varepsilon_{A} \varepsilon_{B} \varepsilon_{A B}=1$ where $\varepsilon_{A}=\operatorname{sgn}(\operatorname{tr}(A)), \varepsilon_{B}=\operatorname{sgn}(\operatorname{tr}(B))$ and $\varepsilon_{A B}=\operatorname{sgn}(\operatorname{tr}(A B))$.

Proof. Without loss of generality, we may assume the axes of $A$ and $B$ are located as in Figure 2. Then

$$
A=\frac{1}{y-x}\left(\begin{array}{cc}
\lambda^{-1} y-\lambda x & \left(\lambda-\lambda^{-1}\right) x y \\
-\left(\lambda-\lambda^{-1}\right) & \lambda y-\lambda^{-1} x
\end{array}\right), \quad B=\left(\begin{array}{cc}
\mu^{-1} & 0 \\
0 & \mu
\end{array}\right)
$$

with $\lambda^{2}>1, \mu^{2}>1$ and $x<0<y$. Then

$$
A B=\frac{1}{y-x}\left(\begin{array}{cc}
\left(\lambda^{-1} y-\lambda x\right) \mu^{-1} & \left(\lambda-\lambda^{-1}\right) x y \mu \\
-\left(\lambda-\lambda^{-1}\right) \mu^{-1} & \left(\lambda y-\lambda^{-1} x\right) \mu
\end{array}\right)
$$


and the trace of $A B$ is

$$
\operatorname{tr}(A B)=\frac{1}{y-x}\left\{\left(\lambda^{-1} \mu^{-1}+\lambda \mu\right) y-\left(\lambda \mu^{-1}+\lambda^{-1} \mu\right) x\right\} .
$$

Since $\lambda^{2}>1$ and $\mu^{2}>1$, there are two possibilities for the sign of $\lambda \mu$. Recall that $x<0<y$. If $\lambda \mu>1$, then

$$
\operatorname{tr}(A B)-2=\frac{1}{(y-x) \lambda \mu}\left\{(\lambda \mu-1)^{2} y-(\lambda-\mu)^{2} x\right\}>0 .
$$

If $\lambda \mu<-1$, then

$$
\operatorname{tr}(A B)+2=\frac{1}{(y-x) \lambda \mu}\left\{(\lambda \mu+1)^{2} y-(\lambda+\mu)^{2} x\right\}<0 .
$$

Thus if $\lambda \mu>1\left(\Leftrightarrow \varepsilon_{A} \varepsilon_{B}=1\right)$, then $\operatorname{tr}(A B)>2\left(\Leftrightarrow \varepsilon_{A B}=1\right)$ and if $\lambda \mu<-1(\Leftrightarrow$ $\left.\varepsilon_{A} \varepsilon_{B}=-1\right)$, then $\operatorname{tr}(A B)<-2\left(\Leftrightarrow \varepsilon_{A B}=-1\right)$. Therefore $\varepsilon_{A} \varepsilon_{B} \varepsilon_{A B}=1$.

Theorem 4.9. The Fricke space of a one-holed torus $\Sigma(1,1)$ can be identified with the open subset of $\mathbb{R}^{3}$ such that

$$
\left\{\left(t_{A}, t_{B}, t_{A B}\right) \in(2, \infty)^{3} \mid f_{A B}<-4\right\}
$$

where $f_{A B}=t_{A}^{2}+t_{B}^{2}+t_{A B}^{2}-t_{A} t_{B} t_{A B}-4$.

Proof. By Proposition 4.8 and the various of lifts from $\operatorname{PSL}(2, \mathbb{R})$ to $\mathbf{S L}(2, \mathbb{R})$ in (2), the possible traces of $A, B$ and $A B$ are

$$
\begin{aligned}
\tilde{\mathcal{F}}_{1,1} & \text { let } \\
= & (2, \infty) \times(2, \infty) \times(2, \infty) \bigcup(-\infty,-2) \times(2, \infty) \times(-\infty,-2) \\
\bigcup & (2, \infty) \times(-\infty,-2) \times(-\infty,-2) \bigcup(-\infty,-2) \times(-\infty,-2) \times(2, \infty) .
\end{aligned}
$$

Recall that $\operatorname{tr}(C)=t_{A}^{2}+t_{B}^{2}+t_{A B}^{2}-t_{A} t_{B} t_{A B}-2=f_{A B}+2$. Since the condition for the discreteness of a holonomy group of $\Sigma(1,1)$ is $\operatorname{tr}(C)<-2$, we obtain the condition $f_{A B}=\operatorname{tr}(C)-2<-4$ which is

$$
t_{A}^{2}+t_{B}^{2}+t_{A B}^{2}-t_{A} t_{B} t_{A B}<0 .
$$

Therefore the Fricke space $\mathcal{F}(\Sigma(1,1))=\widetilde{\mathcal{F}}_{1,1} / H^{1}(M ; \mathbb{Z} / 2 \mathbb{Z})$ can be identified with the open subset of $\mathbb{R}^{3}$ as we claimed.

\subsection{Four-holed sphere $\Sigma(0,4)$}

The Fricke space of a four-holed sphere $\Sigma(0,4)$ is more complicated than those of a three-holed sphere $\Sigma(0,3)$ and a one-holed torus $\Sigma(1,1)$. Suppose $\Sigma(0,4)$ is a 4 -holed sphere with boundary components $A_{1}, A_{2}, A_{3}, A_{4}$ subject to the relation

$$
A_{4} A_{3} A_{2} A_{1}=I
$$

Let

$$
A_{5}=A_{1}^{-1} A_{2}^{-1}, A_{6}=A_{2}^{-1} A_{3}^{-1}, A_{7}=A_{1}^{-1} A_{3}^{-1}, \text { and } A_{8}=A_{2}^{-1} A_{4}^{-1} ;
$$


Equivalently we have the relations

$$
A_{5} A_{2} A_{1}=I, A_{6} A_{3} A_{2}=I, A_{7} A_{3} A_{1}=I \text {, and } A_{8} A_{4} A_{2}=I .
$$

The elements $A_{5}, A_{6}, A_{7}, A_{8}$ correspond to simple loops on $\Sigma(0,4)$ which separate $\Sigma(0,4)$ into two 3 -holed spheres. Thus $A_{5}, A_{6}, A_{7}, A_{8}$ called the pants separating elements of $\Sigma(0,4)$. We denote $t_{i}=\operatorname{tr}\left(A_{i}\right)$ and $t_{i j}=\operatorname{tr}\left(A_{i} A_{j}\right)$. Then

$$
t_{5}=t_{12}=t_{34}, t_{6}=t_{23}=t_{14}, t_{7}=t_{13}, \text { and } t_{8}=t_{24}
$$

since $A_{5}=\left(A_{2} A_{1}\right)^{-1}=A_{4} A_{3}$, and $A_{6}=\left(A_{3} A_{2}\right)^{-1}=A_{1} A_{4}$.

We will show the $\mathbf{S L}(2, \mathbb{C})$-character variety of a four-holed sphere $\Sigma(0,4)$ is a six-dimensional hypersurface in $\mathbb{C}^{7}$. First we will show the traces $t_{7}=t_{13}$ and $t_{8}=t_{24}$ are expressed by other six traces.

Proposition 4.10. Let $t_{i}$ and $t_{i j}$ be traces of elements of a holonomy group a four-holed sphere $\Sigma(0,4)$ satisfying $(31)$ and $(32)$. Then the following equations hold.

$$
\begin{aligned}
t_{13}+t_{24}= & t_{1} t_{3}+t_{2} t_{4}-t_{12} t_{23} \stackrel{\text { let }}{=} \beta \\
t_{13} \cdot t_{24}= & \left(t_{1}^{2}+t_{2}^{2}+t_{3}^{2}+t_{4}^{2}\right)+\left(t_{12}^{2}+t_{23}^{2}\right)-\left(t_{1} t_{2}+t_{3} t_{4}\right) t_{12} \\
& -\left(t_{2} t_{3}+t_{1} t_{4}\right) t_{23}+t_{1} t_{2} t_{3} t_{4}-4 \stackrel{\text { let }}{=} \gamma .
\end{aligned}
$$

Proof. To prove above formulas, we apply Trace Identity (23) repeatedly. From $A_{7}=A_{1}^{-1} A_{3}^{-1}=\left(A_{4} A_{3} A_{2}\right) A_{3}^{-1}$, we get

$$
\begin{aligned}
t_{13} & =\operatorname{tr}\left(A_{4} A_{3} A_{2} A_{3}^{-1}\right)=\operatorname{tr}\left(A_{4}\right) \operatorname{tr}\left(A_{3} A_{2} A_{3}^{-1}\right)-\operatorname{tr}\left(A_{4}^{-1} A_{3} A_{2} A_{3}^{-1}\right) \\
& =t_{4} t_{2}-\operatorname{tr}\left(A_{3} A_{2} A_{1} A_{3} A_{2} A_{3}^{-1}\right)=t_{2} t_{4}-\operatorname{tr}\left(A_{2} A_{1} A_{3} A_{2}\right) \\
& =t_{2} t_{4}-\operatorname{tr}\left(A_{2} A_{1}\right) \operatorname{tr}\left(A_{3} A_{2}\right)+\operatorname{tr}\left(A_{1}^{-1} A_{2}^{-1} A_{3} A_{2}\right) \\
& =t_{2} t_{4}-t_{12} t_{23}+\operatorname{tr}\left(A_{1}^{-1}\right) \operatorname{tr}\left(A_{2}^{-1} A_{3} A_{2}\right)-\operatorname{tr}\left(A_{1} A_{2}^{-1} A_{3} A_{2}\right) \\
& =t_{2} t_{4}-t_{12} t_{23}+t_{1} t_{3}-\operatorname{tr}\left(A_{2}^{-1} A_{3} A_{2} A_{1}\right) \\
& =t_{2} t_{4}-t_{12} t_{23}+t_{1} t_{3}-\operatorname{tr}\left(A_{2}^{-1} A_{4}^{-1}\right)=t_{2} t_{4}-t_{12} t_{23}+t_{1} t_{3}-t_{24} .
\end{aligned}
$$

Since $t_{13} \cdot t_{24}=\operatorname{tr}\left(A_{7}\right) \operatorname{tr}\left(A_{8}\right)=\operatorname{tr}\left(A_{7} A_{8}\right)+\operatorname{tr}\left(A_{7}^{-1} A_{8}\right)$, we compute $\operatorname{tr}\left(A_{7} A_{8}\right)$ and $\operatorname{tr}\left(A_{7}^{-1} A_{8}\right)$. From $A_{7}=\left(A_{3} A_{1}\right)^{-1}$ and $A_{8}=\left(A_{4} A_{2}\right)^{-1}$, we have

$$
\begin{aligned}
\operatorname{tr}\left(A_{7} A_{8}\right) & =\operatorname{tr}\left(A_{8}^{-1} A_{7}^{-1}\right)=\operatorname{tr}\left(A_{4} A_{2} A_{3} A_{1}\right)=\operatorname{tr}\left(\left(A_{3} A_{2} A_{1}\right)^{-1} A_{2} A_{3} A_{1}\right) \\
& =\operatorname{tr}\left(A_{1}^{-1} A_{2}^{-1} A_{3}^{-1} A_{2} A_{3} A_{1}\right)=\operatorname{tr}\left(A_{2}^{-1} A_{3}^{-1} A_{2} A_{3}\right) \\
& =t_{2}^{2}+t_{3}^{2}+t_{23}^{2}-t_{2} t_{3} t_{23}-2
\end{aligned}
$$

by the commutator equation (25).

$$
\begin{aligned}
\operatorname{tr}\left(A_{7}^{-1} A_{8}\right) & =\operatorname{tr}\left(A_{3} A_{1} A_{2}^{-1} A_{4}^{-1}\right)=\operatorname{tr}\left(A_{3} A_{1} A_{2}^{-1} A_{3} A_{2} A_{1}\right) \\
& =\operatorname{tr}\left(A_{3} A_{1} A_{2}^{-1}\right) \operatorname{tr}\left(A_{3} A_{2} A_{1}\right)-\operatorname{tr}\left(A_{2} A_{1}^{-1} A_{3}^{-1} A_{3} A_{2} A_{1}\right) \\
& =\operatorname{tr}\left(A_{3} A_{1} A_{2}^{-1}\right) \operatorname{tr}\left(A_{4}^{-1}\right)-\operatorname{tr}\left(A_{2} A_{1}^{-1} A_{2} A_{1}\right) \\
& =\left\{\operatorname{tr}\left(A_{3}\right) \operatorname{tr}\left(A_{1} A_{2}^{-1}\right)-\operatorname{tr}\left(A_{3}^{-1} A_{1} A_{2}^{-1}\right)\right\} t_{4}-\operatorname{tr}\left(A_{2} A_{1}^{-1} A_{2} A_{1}\right)
\end{aligned}
$$




$$
\begin{aligned}
= & \left\{t_{3}\left(t_{1} t_{2}-t_{12}\right)-\operatorname{tr}\left(A_{2}^{-1} A_{3}^{-1} A_{1}\right)\right\} t_{4}-\operatorname{tr}\left(A_{2} A_{1}^{-1} A_{2} A_{1}\right) \\
= & \left\{t_{3}\left(t_{1} t_{2}-t_{12}\right)-\operatorname{tr}\left(A_{2}^{-1} A_{3}^{-1}\right) \operatorname{tr}\left(A_{1}\right)+\operatorname{tr}\left(A_{3} A_{2} A_{1}\right)\right\} t_{4} \\
& -\operatorname{tr}\left(A_{2} A_{1}^{-1}\right) \operatorname{tr}\left(A_{2} A_{1}\right)+\operatorname{tr}\left(A_{1} A_{2}^{-1} A_{2} A_{1}\right) \\
= & \left\{t_{3}\left(t_{1} t_{2}-t_{12}\right)-t_{23} t_{1}+t_{4}\right\} t_{4}-\left(t_{2} t_{1}-t_{12}\right) t_{12}+\left(t_{1}^{2}-2\right) \\
= & t_{1} t_{2} t_{3} t_{4}-t_{3} t_{4} t_{12}-t_{1} t_{4} t_{23}+t_{4}^{2}-t_{1} t_{2} t_{12}+t_{12}^{2}+t_{1}^{2}-2 .
\end{aligned}
$$

Therefore we obtain $t_{13} \cdot t_{24}=\operatorname{tr}\left(A_{7} A_{8}\right)+\operatorname{tr}\left(A_{7}^{-1} A_{8}\right)=\left(t_{1}^{2}+t_{2}^{2}+t_{3}^{2}+t_{4}^{2}\right)+$ $\left(t_{12}^{2}+t_{23}^{2}\right)-\left(t_{1} t_{2}+t_{3} t_{4}\right) t_{12}-\left(t_{2} t_{3}+t_{1} t_{4}\right) t_{23}+t_{1} t_{2} t_{3} t_{4}-4$.

Above Proposition 4.10 implies that the traces $t_{13}$ and $t_{24}$ are roots of the quadratic equation

$$
z^{2}-\beta z+\gamma=0
$$

where the coefficients $\beta$ and $\gamma$ are from (34) and (35). Thus we have

$$
t_{13}, t_{24}=\frac{\beta \pm \sqrt{\beta^{2}-4 \gamma}}{2} \text {. }
$$

But we do not know $t_{13}$ is $\frac{\beta+\sqrt{\beta^{2}-4 \gamma}}{2}$ or $\frac{\beta-\sqrt{\beta^{2}-4 \gamma}}{2}$, because it is possible to happen all three cases $t_{13}<t_{24}, t_{13}=t_{24}$, and $t_{13}>t_{24}$.

From Equations (34) and (35), we can remove $t_{24}$. Since

$$
t_{24}=t_{1} t_{3}+t_{2} t_{4}-t_{12} t_{23}-t_{13},
$$

we obtain

$$
\begin{aligned}
& t_{13}\left(t_{1} t_{3}+t_{2} t_{4}-t_{12} t_{23}-t_{13}\right)=\left(t_{1}^{2}+t_{2}^{2}+t_{3}^{2}+t_{4}^{2}\right)+\left(t_{12}^{2}+t_{23}^{2}\right) \\
& \quad-\left(t_{1} t_{2}+t_{3} t_{4}\right) t_{12}-\left(t_{2} t_{3}+t_{1} t_{4}\right) t_{23}+t_{1} t_{2} t_{3} t_{4}-4
\end{aligned}
$$

Therefore the $\mathbf{S L}(2, \mathbb{C})$-character variety of a four-holed sphere $\Sigma(0,4)$ is the hypersurface in $\mathbb{C}^{7}$ satisfying

$$
\begin{aligned}
& \left(t_{1}^{2}+t_{2}^{2}+t_{3}^{2}+t_{4}^{2}\right)+\left(t_{12}^{2}+t_{23}^{2}+t_{13}^{2}\right)+t_{12} t_{23} t_{13}+t_{1} t_{2} t_{3} t_{4}-4 \\
& \quad-\left(t_{1} t_{2}+t_{3} t_{4}\right) t_{12}-\left(t_{2} t_{3}+t_{1} t_{4}\right) t_{23}-\left(t_{1} t_{3}+t_{2} t_{4}\right) t_{13}=0
\end{aligned}
$$

for $\left(t_{1}, t_{2}, t_{3}, t_{4}, t_{12}, t_{23}, t_{13}\right) \in \mathbb{C}^{7}$.

Suppose a four-holed sphere $\Sigma(0,4)$ is equipped with a hyperbolic structure. Then a holonomy group with redundant relations

$$
\Gamma=\left\langle A_{1}, A_{2}, A_{3}, A_{4}, A_{5}, A_{6} \mid A_{4} A_{3} A_{2} A_{1}=I, A_{5} A_{2} A_{1}=I, A_{6} A_{3} A_{2}=I\right\rangle
$$

of $\Sigma(0,4)$ is discrete if and only if the axes of $A_{1}, A_{2}, A_{3}, A_{4}, A_{5}, A_{6}$ are located as in Figure 3 up to conjugation.

Theorem 4.11. Suppose $A_{1}, A_{2}, A_{3}, A_{4}, A_{5}, A_{6} \in \mathbf{S L}(2, \mathbb{R})$ are hyperbolic elements such that $A_{4} A_{3} A_{2} A_{1}=I, A_{5} A_{2} A_{1}=I$, and $A_{6} A_{3} A_{2}=I$. Let $\xi_{j}$ be the involution corresponding to $A_{j}$ and $\varepsilon_{j}=\operatorname{sgn}\left(\operatorname{tr}\left(A_{j}\right)\right)$. Then

$$
\left\langle\xi_{1}, \xi_{2}\right\rangle>1,\left\langle\xi_{1}, \xi_{3}\right\rangle>1,\left\langle\xi_{1}, \xi_{4}\right\rangle>1,\left\langle\xi_{1}, \xi_{5}\right\rangle>1,\left\langle\xi_{1}, \xi_{6}\right\rangle<-1,
$$




$$
\begin{aligned}
& \left\langle\xi_{2}, \xi_{3}\right\rangle>1,\left\langle\xi_{2}, \xi_{4}\right\rangle>1,\left\langle\xi_{2}, \xi_{5}\right\rangle>1,\left\langle\xi_{2}, \xi_{6}\right\rangle>1, \\
& \left\langle\xi_{3}, \xi_{4}\right\rangle>1,\left\langle\xi_{3}, \xi_{5}\right\rangle<-1,\left\langle\xi_{3}, \xi_{6}\right\rangle>1 \\
& \left\langle\xi_{4}, \xi_{5}\right\rangle<-1,\left\langle\xi_{4}, \xi_{6}\right\rangle<-1 \\
& \left\langle\xi_{5}, \xi_{6}\right\rangle \in(-1,1)
\end{aligned}
$$

if and only if

$$
\begin{gathered}
\varepsilon_{1} \varepsilon_{2} \varepsilon_{3} \varepsilon_{4}=1, \varepsilon_{1} \varepsilon_{2} \varepsilon_{5}=-1, \varepsilon_{2} \varepsilon_{3} \varepsilon_{6}=-1, \\
f_{56}=t_{5}^{2}+t_{6}^{2}+t_{56}^{2}-t_{5} t_{6} t_{56}-4<0 .
\end{gathered}
$$

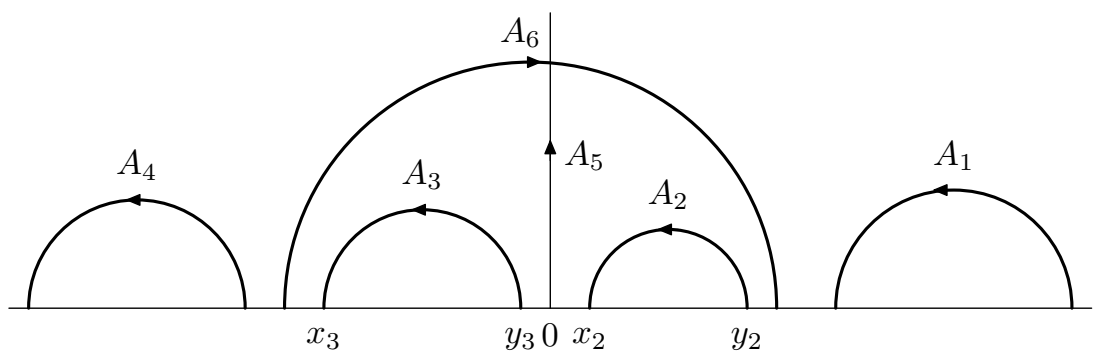

Figure 3 . The locations of axes $A_{1}, A_{2}, A_{3}, A_{4}, A_{5}, A_{6}$ with $A_{4} A_{3} A_{2} A_{1}=I, A_{5} A_{2} A_{1}=I$ and $A_{6} A_{3} A_{2}=I$.

Proof. Notice that

$$
A_{4} A_{3} A_{5}^{-1}=A_{4} A_{3} A_{2} A_{1}=I \text {, and } A_{1} A_{4} A_{6}^{-1}=A_{1} A_{4} A_{3} A_{2}=I .
$$

And we know $\left\langle\xi_{5}, \xi_{6}\right\rangle \in(-1,1)$ is equivalent to $f_{56}<0$ by Corollary 3.11-(3).

$(\Rightarrow)$ By Theorem 4.1 we have $\varepsilon_{1} \varepsilon_{2} \varepsilon_{5}=-1$ and $\varepsilon_{2} \varepsilon_{3} \varepsilon_{6}=-1$ since $A_{5} A_{2} A_{1}=$ $I,\left\langle\xi_{1}, \xi_{2}\right\rangle>1,\left\langle\xi_{2}, \xi_{5}\right\rangle>1,\left\langle\xi_{1}, \xi_{5}\right\rangle>1$ and $A_{6} A_{3} A_{2}=I,\left\langle\xi_{2}, \xi_{3}\right\rangle>1,\left\langle\xi_{2}, \xi_{6}\right\rangle>$ $1,\left\langle\xi_{3}, \xi_{6}\right\rangle>1$ respectively. From $A_{4} A_{3} A_{5}^{-1}=I$ and $\xi_{A^{-1}}=-\xi_{A}$, we derive $\left\langle\xi_{3}, \xi_{4}\right\rangle>1,\left\langle\xi_{3},-\xi_{5}\right\rangle>1$ and $\left\langle\xi_{4},-\xi_{5}\right\rangle>1$. Thus $\varepsilon_{5} \varepsilon_{3} \varepsilon_{4}=-1$. Therefore $\varepsilon_{1} \varepsilon_{2} \varepsilon_{3} \varepsilon_{4}=\left(\varepsilon_{1} \varepsilon_{2} \varepsilon_{5}\right)\left(\varepsilon_{5} \varepsilon_{3} \varepsilon_{4}\right)=(-1)^{2}=1$.

$(\Leftarrow)$ Since $\varepsilon_{1} \varepsilon_{2} \varepsilon_{3} \varepsilon_{4}=1, \varepsilon_{1} \varepsilon_{2} \varepsilon_{5}=-1, \varepsilon_{2} \varepsilon_{3} \varepsilon_{6}=-1$, and $\varepsilon_{i}^{2}=1$, we induce $\varepsilon_{5} \varepsilon_{3} \varepsilon_{4}=-1$ and $\varepsilon_{6} \varepsilon_{4} \varepsilon_{1}=-1$. Since $A_{4} A_{3} A_{5}^{-1}=I, A_{5} A_{2} A_{1}=I, A_{1} A_{4} A_{6}^{-1}=$ $I, A_{6} A_{3} A_{2}=I$ and $f_{56}<0$, we obtain every inequalities except $\left\langle\xi_{1}, \xi_{3}\right\rangle>1$ and $\left\langle\xi_{2}, \xi_{4}\right\rangle>1$. We claim that if $\left\langle\xi_{1}, \xi_{5}\right\rangle>1,\left\langle\xi_{2}, \xi_{3}\right\rangle>1$ and $\left\langle\xi_{3}, \xi_{5}\right\rangle<-1$, then $\left\langle\xi_{1}, \xi_{3}\right\rangle>1$. Without loss of generality, we assume the axis $\ell_{1}$ of $A_{1}$ is contained the half-plane $\mathcal{H}_{\ell_{5}}$ of $A_{5}$. If the axis $\ell_{3}$ is contained in $\mathcal{H}_{\ell_{5}}$, then it contradicts for the axes $\ell_{2}$ and $\ell_{3}$ are the same direction. Thus the axis $\ell_{3}$ should be contained in $\mathcal{H}_{\ell_{5}}^{c}$. Since $\left\langle\xi_{1}, \xi_{5}\right\rangle>1$ and $\left\langle\xi_{3}, \xi_{5}\right\rangle<-1$, we have $\left\langle\xi_{1}, \xi_{3}\right\rangle>1$ (i.e., $\ell_{1}$ and $\ell_{3}$ are separated the same direction) by Theorem 3.8(1) and (2). Similarly we can show $\left\langle\xi_{2}, \xi_{4}\right\rangle>1$. 
Since $f_{56}<0$ is one of the conditions for the discreteness for a holonomy group of $\Sigma(0,4)$, we need to know about $f_{56}=t_{5}^{2}+t_{6}^{2}+t_{56}^{2}-t_{5} t_{6} t_{56}-4$. Recall $t_{5}=t_{12}$ and $t_{6}=t_{23}$. First we calculate $t_{56}$.

$$
\begin{aligned}
t_{56} & =\operatorname{tr}\left(A_{5} A_{6}\right)=\operatorname{tr}\left(A_{6}^{-1} A_{5}^{-1}\right)=\operatorname{tr}\left(A_{3} A_{2} A_{2} A_{1}\right) \\
& =\operatorname{tr}\left(A_{2} A_{2} A_{1} A_{3}\right)=\operatorname{tr}\left(A_{2}\right) \operatorname{tr}\left(A_{2} A_{1} A_{3}\right)-\operatorname{tr}\left(A_{2}^{-1} A_{2} A_{1} A_{3}\right) \\
& =t_{2} \operatorname{tr}\left(A_{3} A_{2} A_{1}\right)-\operatorname{tr}\left(A_{1} A_{3}\right)=t_{2} \operatorname{tr}\left(A_{4}^{-1}\right)-t_{13}=t_{2} t_{4}-t_{13} .
\end{aligned}
$$

Thus

$$
\begin{aligned}
f_{56} & =t_{12}^{2}+t_{23}^{2}+\left(t_{2} t_{4}-t_{13}\right)^{2}-t_{12} t_{23}\left(t_{2} t_{4}-t_{13}\right)-4 \\
& =t_{12}^{2}+t_{23}^{2}+t_{2}^{2} t_{4}^{4}-2 t_{2} t_{4} t_{13}+t_{13}^{2}-t_{2} t_{4} t_{12} t_{23}+t_{12} t_{23} t_{13}-4 \\
& =\left(t_{12}^{2}+t_{23}^{2}+t_{13}^{2}+t_{12} t_{23} t_{13}-4\right)+t_{2}^{2} t_{4}^{4}-2 t_{2} t_{4} t_{13}-t_{2} t_{4} t_{12} t_{23} .
\end{aligned}
$$

Hence we need the trace $t_{13}$ to express the Fricke space of a four-holed sphere.

Theorem 4.12. The Fricke space of a four-holed sphere $\Sigma(0,4)$ can be identified with the hypersurface of $\mathbb{R}^{7}$ such that

$$
\left\{\left(t_{1}, t_{2}, t_{3}, t_{4}, t_{12}, t_{23}, t_{13}\right) \in(-\infty,-2)^{7} \mid h_{0,4}=0, f_{56}<0\right\}
$$

where

$$
\begin{aligned}
(39) \quad h_{0,4}= & \left(t_{1}^{2}+t_{2}^{2}+t_{3}^{2}+t_{4}^{2}\right)+\left(t_{12}^{2}+t_{23}^{2}+t_{13}^{2}\right)+t_{12} t_{23} t_{13}+t_{1} t_{2} t_{3} t_{4}-4 \\
& -\left(t_{1} t_{2}+t_{3} t_{4}\right) t_{12}-\left(t_{2} t_{3}+t_{1} t_{4}\right) t_{23}-\left(t_{1} t_{3}+t_{2} t_{4}\right) t_{13} \\
(40) \quad f_{56}= & \left(t_{12}^{2}+t_{23}^{2}+t_{13}^{2}+t_{12} t_{23} t_{13}-4\right)+t_{2}^{2} t_{4}^{4}-2 t_{2} t_{4} t_{13}-t_{2} t_{4} t_{12} t_{23} .
\end{aligned}
$$

Proof. Since the fundamental group of $M=\Sigma(0,4)$ is a free group of rank 3 , $H^{1}(M ; \mathbb{Z} / 2 \mathbb{Z}) \cong \operatorname{Hom}\left(\pi_{1}(M), \mathbb{Z} / 2 \mathbb{Z}\right)$ is isomorphic to $\mathbb{Z} / 2 \mathbb{Z} \oplus \mathbb{Z} / 2 \mathbb{Z} \oplus \mathbb{Z} / 2 \mathbb{Z}$. Thus if $\left\{A_{1}, A_{2}, A_{3}, A_{4}\right\}$ is a lifted $\mathbf{S L}(2, \mathbb{C})$-representation of $\Sigma(0,4)$, then

$$
\begin{aligned}
& \left\{A_{1}, A_{2},-A_{3},-A_{4}\right\},\left\{A_{1},-A_{2}, A_{3},-A_{4}\right\},\left\{A_{1},-A_{2},-A_{3}, A_{4}\right\}, \\
& \left\{-A_{1}, A_{2}, A_{3},-A_{4}\right\},\left\{-A_{1}, A_{2},-A_{3}, A_{4}\right\},\left\{-A_{1},-A_{2}, A_{3}, A_{4}\right\}, \\
& \text { and }\left\{-A_{1},-A_{2},-A_{3},-A_{4}\right\}
\end{aligned}
$$

are other liftable $\mathbf{S L}(2, \mathbb{C})$-representations. By Theorem 4.11 , the signs of elements of a discrete holonomy group $\Gamma$ of $\Sigma(0,4)$ satisfy relations

$$
\varepsilon_{1} \varepsilon_{2} \varepsilon_{3} \varepsilon_{4}=1, \varepsilon_{1} \varepsilon_{2} \varepsilon_{5}=\varepsilon_{1} \varepsilon_{2} \varepsilon_{12}=-1 \text {, and } \varepsilon_{2} \varepsilon_{3} \varepsilon_{6}=\varepsilon_{2} \varepsilon_{3} \varepsilon_{23}=-1 \text {. }
$$

And the discreteness of $\Gamma$ ensures a pants separating element $A_{7}=A_{1}^{-1} A_{3}^{-1}$ induces another relation

$$
\varepsilon_{1} \varepsilon_{3} \varepsilon_{7}=\varepsilon_{1} \varepsilon_{3} \varepsilon_{13}=-1 .
$$

Thus the possible signs $\left(\varepsilon_{1}, \varepsilon_{2}, \varepsilon_{3}, \varepsilon_{4}, \varepsilon_{12}, \varepsilon_{23}, \varepsilon_{13}\right)$ of traces are

$$
\begin{aligned}
& (+,+,+,+,-,-,-),(+,+,-,-,-,+,+),(+,-,+,-,+,+,-), \\
& (+,-,-,+,+,-,+),(-,+,+,-,+,-,+),(-,+,-,+,+,+,-), \\
& (-,-,+,+,-,+,+), \text { and }(-,-,-,-,-,-,-) .
\end{aligned}
$$


By the quotient action of $H^{1}(M ; \mathbb{Z} / 2 \mathbb{Z}) \cong \mathbb{Z} / 2 \mathbb{Z} \oplus \mathbb{Z} / 2 \mathbb{Z} \oplus \mathbb{Z} / 2 \mathbb{Z}$, the Fricke space $\mathcal{F}(\Sigma(0,4))$ can be considered as a subset of $(-\infty,-2)^{7}$. Since the $\mathbf{S L}(2, \mathbb{C})$ character variety of $\Sigma(0,4)$ satisfies Equation $(36)$ which is $h_{0,4}=0$ and the discreteness of $\Gamma$ requires the condition $f_{56}<0$, the Fricke space $\mathcal{F}(\Sigma(0,4))$ is identified with the six-dimensional hypersurface of $\mathbb{R}^{7}$ as we claimed.

Acknowledgements. I would like to thank W. Goldman for introducing me to this subject. I also thank the referee for pointing out some errors in an earlier version of this paper.

\section{References}

[1] L. Bers and F. Gardiner, Fricke spaces, Adv. in Math. 62 (1986), no. 3, 249-284.

[2] M. Culler, Lifting representations to covering groups, Adv. in Math. 59 (1986), no. 1, 64-70.

[3] W. Goldman, The symplectic nature of fundamental groups of surfaces, Adv. in Math. 54 (1984), no. 2, 200-225.

[4] - Topological components of spaces of representations, Invent. Math. 93 (1988), no. 3, 557-607.

[5] _ Convex real projective structures on compact surfaces, J. Differential Geom. 31 (1990), no. 3, 791-845.

[6] _ Locally homogeneous geometric manifolds, Proceedings of the International Congress of Mathematicians. Volume II, 717-744, Hindustan Book Agency, New Delhi, 2010.

[7] H. C. Kim, Embedding of the Teichmüller Space into Goldman Space, J. Korean Math. Soc. 43 (2006), no. 6, 1231-1252.

[8] N. Kuiper, On convex locally projective spaces, Convegno Internazionale di Geometria Differenziale, Italia, 1953, pp. 200-213. Edizioni Cremonese, Roma, 1954.

[9] W. Magnus, Rings of Fricke characters and automorphism groups of free groups, Math. Z. 170 (1980), no. 1, 91-103.

[10] K. Matsuzaki and M. Taniguchi, Hyperbolic Manifolds and Kleinian Groups, Oxford Science Publications, Oxford University Press, 1998.

[11] J. Morgan and P. Shalen, Degenerations of hyperbolic structures. III. Actions of 3manifold groups on trees and Thurston's compactness theorem, Ann. of Math. (2) 127 (1988), no. 3, 457-519.

[12] C. Procesi, The invariant theory of $n \times n$ matrices, Adv. in Math. 19 (1976), no. 3, 306-381.

[13] J. Ratcliffe, Foundations of Hyperbolic Manifolds, Graduate Texts in Mathematics 149, Springer, 1994

[14] D. Sullivan and W. Thurston, Manifolds with canonical coordinates: some examples, Enseign. Math. 29 (1983), no. 1-2, 15-25.

[15] A. Weil, On discrete subgroups of Lie groups I, Ann. of Math. (2) 72 (1960), 369-384.

Department of Mathematics Education

Korea University

Seoul 136-701, KoreA

E-mail address: hongchan@korea.ac.kr 